\title{
Pseudospin modulation in coupled graphene systems
}

\author{
Chen-Di Han $\odot,{ }^{1}$ Hong-Ya Xu, ${ }^{1}$ and Ying-Cheng Lai $\odot^{1,2, *}$ \\ ${ }^{1}$ School of Electrical, Computer and Energy Engineering, Arizona State University, Tempe, Arizona 85287, USA \\ ${ }^{2}$ Department of Physics, Arizona State University, Tempe, Arizona 85287, USA
}

(Received 4 May 2020; revised 5 August 2020; accepted 10 August 2020; published 14 September 2020)

\begin{abstract}
The ability to manipulate pseudospin can find applications in Dirac-material based spintronics. Unlike the transport of real spin that can be modulated by a magnetic field, some form of magnetization, or a spin-transfer torque, pseudospin does not respond to a magnetic field, making modulating pseudospin transport a challenging task. We articulate an asymmetrically coupled cavity-waveguide configuration in graphene and uncover a phenomenon: making the classical dynamics of the cavity deformed can effectively modulate and enhance pseudospin polarization in the waveguide. The underlying mechanism of this remarkable phenomenon can be attributed to chaos-assisted tunneling, which has been well documented in nonrelativistic quantum systems but not yet in Dirac material systems. The finding establishes the feasibility to develop pseudospin modulators for graphene systems through externally applied electrical potential only, with fidelity over $10 \%$ at the effective distance of several cavity sizes along the waveguide.
\end{abstract}

DOI: 10.1103/PhysRevResearch.2.033406

\section{INTRODUCTION}

Graphene and two-dimensional (2D) Dirac materials possess a unique quantum number-pseudospin [1-5]. In each unit cell, there are two nonequivalent atomic sites, so the entire honeycomb lattice can be regarded as constituting two triangular lattices: $\mathrm{A}$ and $\mathrm{B}$, giving rise to two independent possibilities of quasiparticle motions: those confined to $\mathrm{A}$ and $\mathrm{B}$, respectively. Because of the existence of the two alternatives, the quasiparticles are said to have pseudospin-1/2, where two spinor components governed by the Dirac equation are required to describe the quasiparticles. The band structure of the low energy excitations consists of a pair of vertextouching Dirac cones. The pseudospin quantum number can be exploited for storing and transferring information with applications in Dirac material based spintronics [6-9].

Pseudospin, after all, is not the real spin. Usually, the transport of an electron's real spin can be modulated by a magnetic field [10] or some form of magnetization [11,12]. The degree of real spin polarization can also be modulated through the generation of a spin-transfer torque [13,14]. However, pseudospin does not respond to a magnetic field. For graphene, one approach to modulating pseudospin is through mechanical deformation that generates a "pseudomagnetic" field $[9,15,16]$. In another method, an electrical potential induced mass type of perturbation in bilayer or monolayer graphene [6,17-19] leading to an energy gap was used to

\footnotetext{
*Ying-Cheng.Lai@asu.edu

Published by the American Physical Society under the terms of the Creative Commons Attribution 4.0 International license. Further distribution of this work must maintain attribution to the author(s) and the published article's title, journal citation, and DOI.
}

break the sublattice symmetry and induce pseudospin polarization. To uncover alternative methods and physical mechanisms to induce/modulate/control the pseudospin degree of freedom can be of considerable applied value to spintronics.

In this paper, we uncover a mechanism to modulate pseudospin. Focusing on graphene, we exploit the principle of mutual coupling between a waveguide and a nearby cavity. The idea is originated from optics, where the asymmetric configuration of coupled waveguide and microcavity is commonly used to induce Fano resonances in various optical devices [20-23]. Figure 1 illustrates such a transport system, where the graphene waveguide, a channel-like structure through which pseudospin-1/2 quasiparticles propagate, can be fabricated by applying some properly designed electrical potential [24-27] and has been experimentally realized [28,29]. Experimentally, a dielectric cavity in graphene can also be created through an external gate potential [30-34], as in scattering [35-38]. In our work, both circular and deformed cavities are considered, where for the latter, the shape of Africa is chosen $[39,40]$. Due to the proximity of the waveguide and cavity, there is an interaction between them, forming bonding and antibonding states and effectively generating an artificial molecular structure on graphene [41,42].

Due to the presence of the cavity, in the plot of the transmission coefficient through the waveguide versus the electron energy, there are dips, which are the manifestations of Fano resonances. Associated with a resonant mode in the cavity, pseudospin polarization of quasiparticles in the waveguide can emerge. The difficulty with a circular cavity is that the Fano resonances are pronounced, thereby diminishing the transmission through the waveguide. However, we find that, for the deformed cavity, not only can strong pseudospin polarization arise, but large transmission can also be achieved, thanks to the mechanism of chaos-assisted tunneling. Two issues, the fidelity versus the input energy and the effect of distance 


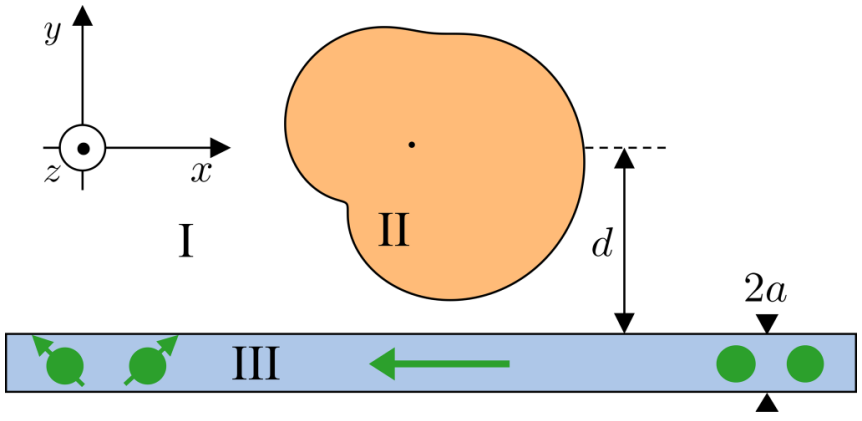

FIG. 1. Coupled cavity-waveguide system in graphene. The upper boundary of the waveguide and the boundary of the cavity divide the relevant space into three regions: I, II, and III. The cavity has the shape of Africa. A beam of pseudospin unpolarized quasiparticles is injected from the right. After passing the interacting region with the cavity, a pseudospin polarized current will emerge.

between the cavity and waveguide, are also addressed, which are relevant to the experimental quantities of spin injection efficiency and diffusion length [8], respectively. We stress that, while the phenomenon of chaos-assisted tunneling has been well documented in nonrelativistic quantum and optical systems [43-56], prior to our work, it had not been uncovered in relativistic quantum transport systems. The overall message is that a deformed cavity geometry can play a beneficial role in modulating pseudospin.

Qualitatively, the mechanism for pseudospin modulation can be understood, as follows. In an integrable cavity, different modes carry different angular momentum. The coupling between the waveguide eigenmode and the cavity becomes strong only for some discrete energy values, so the momentum transfer rate is low for most frequencies. At the resonance frequencies, the symmetry possessed by the integrable cavity would weaken the polarization through some cancellation effect. In addition, the strong confinement in the cavity means weak transmission in the waveguide. For a deformed cavity, the Fano resonances are associated with angular momentum in a broad range, and momentum transfer is the result of both spatial coupling and deformation-induced broadening of the resonances. The collective influences of these factors lead to strong transmission in the waveguide with enhanced pseudospin polarization. A similar phenomenon occurred with chaos assisted momentum transfer in optics [57].

\section{COUPLED WAVEGUIDE-CAVITY GRAPHENE SYSTEM AND FANO RESONANCES}

\section{A. System description and solution method}

The structure of coupled cavity and waveguide is common in optics [20-23], which can be realized through lattice impurities or differential dielectric materials with different values of the refractive index [57,58]. In Dirac material systems, a coupled cavity-waveguide system can be realized with a graphene-metal or graphene-silicon hybrid structure [59,60].

We focus on the graphene based, coupled cavitywaveguide systems as illustrated in Fig. 1, which can be realized by applying an external potentials in different regions of the graphene sheet. To create a waveguide, one can apply an electrical [24] or a magnetic [26] potential. Mathematically, the potential can be smooth, with its profile being some kind of special functions $[25,27]$. Let $x$ be the direction of propagation of pseudospin-1/2 quasiparticles in the graphene waveguide. Effectively, the waveguide is a potential well of finite size in the $y$ direction and infinite in the $x$ direction. For high incoming energy (large wave vector inside), multiple confinement modes inside the waveguide can arise, as has been studied experimentally [28,29]. In the low-energy regime, single mode operation of the graphene waveguide can be realized [61], on which our present study focuses. Likewise, a cavity in a graphene sheet acts as a quantum dot with quantized energy levels. The case of a circular cavity has been previously studied [35-38], which can be realized experimentally with scanning tunneling microscope (STM) tips or through substrate engineering [30-32,34].

For a single valley, the Hamiltonian for the coupled cavitywaveguide system is

$$
H=v_{g} \boldsymbol{\sigma} \cdot \mathbf{p}+U_{\mathrm{II}}(\mathbf{r})+U_{\mathrm{III}}(\mathbf{r})
$$

where $v_{g}$ is the Fermi velocity, $U_{\mathrm{II}}$ and $U_{\mathrm{III}}$ are the cavity and waveguide potential functions, respectively. For simplicity, we assume that the potential profiles are described by the step functions:

$$
\begin{gathered}
U_{\mathrm{II}}(\mathbf{r})= \begin{cases}\hbar v_{g} V_{2}, & \mathbf{r} \in \mathbb{D}_{\mathrm{II}}, \\
0, & \mathbf{r} \notin \mathbb{D}_{\mathrm{II}}\end{cases} \\
U_{\mathrm{III}}(\mathbf{r})= \begin{cases}\hbar v_{g} V_{3}, & \mathbf{r} \in \mathbb{D}_{\mathrm{III}} \\
0, & \mathbf{r} \notin \mathbb{D}_{\mathrm{III}}\end{cases}
\end{gathered}
$$

where $V_{2}$ and $V_{3}$ are the heights of the potential applied to regions II and III, respectively. Due to the presence of the waveguide, even for a circular cavity, the pseudospin-1/2 wave scattering problem cannot be solved analytically. To calculate the basic quantities characterizing the transport, e.g., the transmission and reflection coefficients, we exploit the multiple multipole (MMP) method originated from optics [62-66] and adopted to photonic crystal waveguides [67] and more recently to Dirac-Weyl spinor systems under various circumstances [68-70]. Specifically, without the waveguide, for a single circular cavity on a graphene sheet, associated with various resonant states the angular momenta are conserved, which can be calculated analytically (Appendix A). Likewise, without the cavity, transport through an infinite graphene waveguide can also be solved analytically [24] (Appendix B). For propagation in a given direction inside the graphene waveguide of a finite length, the confinement modes can be obtained with the MMP method. The main source of error is the finite waveguide cutoff in the propagation direction, which can be reduced by increasing the length. For the full system of graphene cavity and waveguide, distinct sets of fictitious poles can be placed inside and outside the cavity and waveguide, and the scattering wave function is given by a linear combination of the contributions from corresponding sets of poles, which can be solved as a pseudoinverse problem (Appendices C and D). 


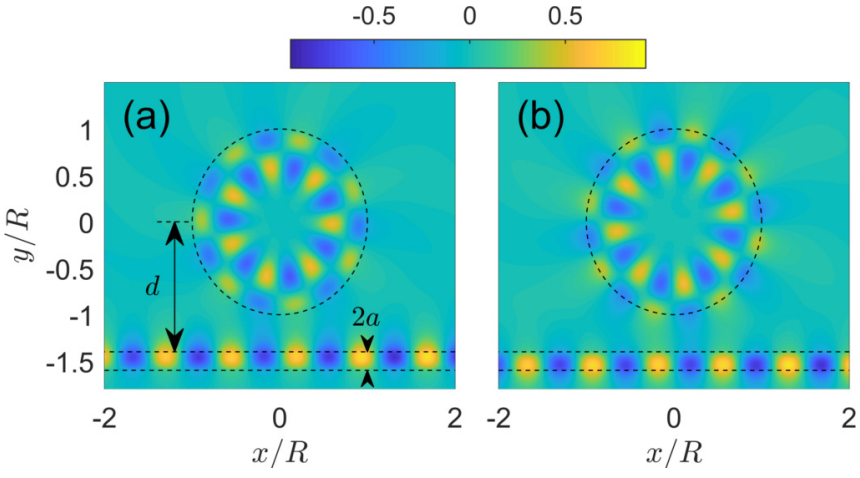

FIG. 2. Wave function patterns in a system where a waveguide is coupled to a circular cavity. (a) For a waveguide of width $2 a$ coupled with a circular cavity of radius $R$, color coded value of the real part of the first spinor component $\psi_{1}$. The distance between the center of the circle to the waveguide is $d$. The dimension of the system is $a=0.1 R$ and $d=1.4 R$. The applied electrical potentials are $V_{2} R=$ 10 and $V_{3} R=8$. For wave vector of the incoming quasiparticles set as $k_{x} R=8.4$, the system is near a resonance mode in the cavity with quantum numbers $l=7$ and $m=2$, where $l$ is the angular momentum index and $m$ is the quantum number associated with the wave function in the radial direction. (b) Color-coded plot of the real part of the second spinor component $\psi_{2}$, where there are more nodes in the angular direction, due to the two-component spinor structure for Dirac fermions.

\section{B. Fano resonances}

The energy spectrum of the waveguide is continuous while that of the cavity is discrete. When the two systems are coupled together, Fano resonances [71] can arise [21,72]. For strong coupling, energy loss from the incident wave in the waveguide can occur due to the excitation of the resonant modes in the cavity, reducing the transmission. Two examples of the wave function profiles for the coupled system are shown in Figs. 2(a) and 2(b), respectively. The transmission coefficient $T_{0}$ of the waveguide can be obtained from these wave functions, which can be defined in terms of the current passing through the interaction region. In particular, we have

$$
T_{0} \equiv J_{x}^{\text {out }} / J_{x}^{\text {out-Free }},
$$

where

$$
J_{x}^{\text {out }}=\iint j_{x} d x d y \text { and } \mathbf{j}=\langle\Psi|\boldsymbol{\sigma}| \Psi\rangle .
$$

The integration for $J^{\text {out }}$ is over the waveguide domain beyond the coupling region directly below the cavity. Due to particle conservation, a higher concentration of the wave function in the cavity means a smaller current in the waveguide and, consequently, smaller transmission $T_{0}$. For simplicity, we focus on the regime of a single transverse mode in the waveguide which, for the specific parameter setting $V_{3} R=8$ in our computations, is given by $k_{x} R<11$.

Figure 3 shows, for $V_{2} R=10$ and $V_{3} R=8$, the transmission coefficient $T_{0}$ of the waveguide versus the normalized dimensionless wave vector $k_{x} R$. In the range of $k_{x} R$ values plotted, two Fano resonances arise, as characterized by the two dips in the transmission curve. The resonance profile is approximately symmetry with respect to the center of the dip,
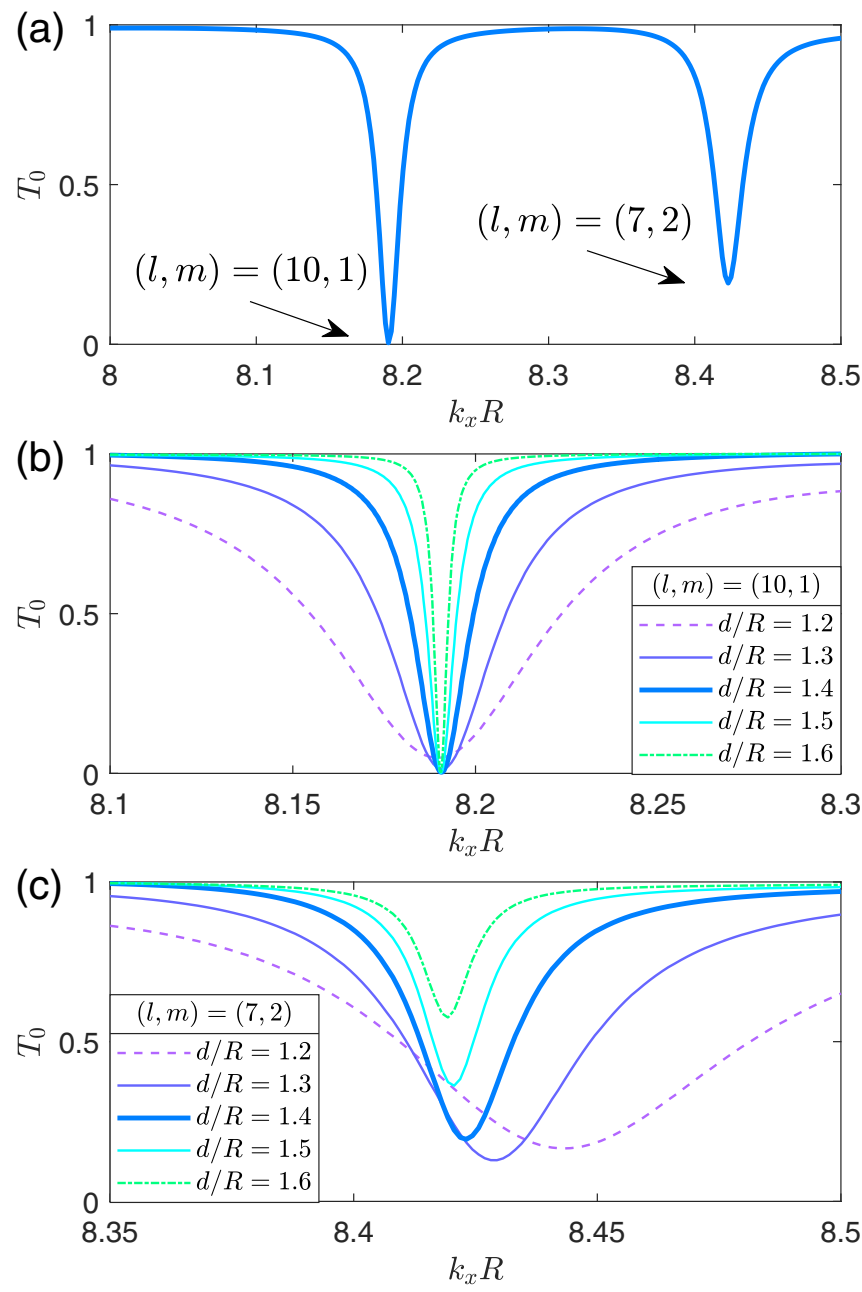

FIG. 3. Representative behavior of transmission $T_{0}$ vs the input wave vector. The parameter setting is $V_{2} R=10$ and $V_{3} R=8$. (a) Two resonant modes as indicated by the corresponding dips in the transmission curve. As the propagating direction in the waveguide is from right to left, the evanescent wave generated leads to a strong coupling with the modes in the cavity with positive angular momentum values $(l>0$, clockwise) only. The two resonant modes are associated with angular momentum quantum numbers $(l, m)=(10,1)$ and $(l, m)=$ $(7,2)$, respectively. [(b) and (c)] For the two resonant modes, respectively, effect of the distance between the waveguide and cavity on the resonance profile. As the distance is increased so that the coupling is weakened, the profile narrows.

signifying its association with a single, distinct mode in the cavity $[42,73]$. The coupling between the propagating mode in the waveguide and a confinement mode in the cavity is enabled through evanescent wave coupling. In the coupling region, the evanescent wave can be expressed in terms of the angular momentum eigenmodes through the Jacobi-Anger expansion as

$$
\begin{aligned}
\chi & =\exp \left[-\alpha_{2}(y+d+a)\right] \exp \left(i k_{x} x\right), \\
& =\sum_{l} n_{l} J_{l}(\epsilon r) \exp (i l \theta),
\end{aligned}
$$

where the geometric parameters $d$ and $a$ are specified in Figs. 1 and $3, n_{l}=\exp \left(-\alpha_{2}(d+a)\right) \exp (i l \phi)$ with 
$\sin \phi=k_{x} /|\epsilon|, \cos \phi=\sqrt{k_{x}^{2}-\epsilon^{2}} /(i|\epsilon|)$ and $\alpha_{2}=\sqrt{k_{x}^{2}-\epsilon^{2}}$, and $\epsilon$ is the total wave vector. As $d$ increases, the exponentially decaying evanescent wave $\chi$ will diminish. The quantity $\phi$ is the angle between $\epsilon$ and the propagating wave vector $k_{x}$, which is imaginary because of the inequality $k_{x}>|\epsilon|$. From the Jacobi-Anger identity, for each momentum value, when $\phi$ is imaginary, the second factor in $n_{l}$ will grow exponentially for positive or negative values of $l$, depending on the sign of $\operatorname{Im}\{\phi\}$, thereby generating a bias in the current. In our setting, the resonant modes in the cavity have $l>0$, which are different from those in elastic scattering where the incident wave is a plane wave (Appendix A).

Intuitively, for a given value of $k_{x}$, the coupling strength between the cavity and waveguide can be defined as [22,73] $C_{s} \equiv 1-T_{0}$. As $k_{x}$ varies, a Fano resonance can arise, where the transmission reaches minimum for certain value of $k_{x}$. Associated with the resonance, coupling is greatly enhanced. The resonance strength can be conveniently characterized by the maximum value of $C_{s}$, which we define as the coupling efficiency for this specific resonance. The width of the resonance is $\Delta k_{x} \equiv k_{x}^{2}-k_{x}^{1}$, where $k_{x}^{1,2}$ are the pair of $k_{x}$ values at which $C_{s}$ is half of its maximum. Another quantity of interest is the frequency shift as induced by the coupling between the cavity and waveguide [74,75], defined as $\delta k \equiv k_{x}^{\max }\left(C_{s}\right)-$ $k_{x}^{\max }(\sigma)$, where $k_{x}^{\max }\left(C_{s}\right)$ is the frequency at the center of the resonance in terms of $C_{s}$ and $k_{x}^{\max }(\sigma)$ is the corresponding quantity in terms of the total scattering cross section $\sigma$. The dimensionless frequency shift is $\delta k R$.

From the results in Fig. 2, we can assess how the characteristics of Fano resonance depend on the cavity-waveguide distance. Intuitively, as the distance increases, the coupling should be weakened. Two representative examples are shown in Fig. 4. For the $(l, m)=(7,2)$ resonance, for small distances the coupling efficiency is large and it decays for large distances, as shown in Fig. 4(a), agreeing with intuition. However, unexpectedly, for the $(l, m)=(10,1)$ mode, the value of the coupling efficiency maintains at unity even for large distances. To understand this surprising behavior, we seek insights from optics, where an optical resonance corresponds to a quasibound state, regardless of the nature of the resonance (e.g., generated by plane wave or through a Fano type of mechanism) $[22,76]$. Associated with a quasibound state, the wave function is characteristically different from that associated with a confinement mode in the cavity which, in fact, can even diverge [23] for $r \rightarrow \infty$ but with an imaginary energy that indicates energy loss. As a result, confinement cannot be maintained indefinitely, which can be characterized by the quality factor $Q$ defined as the ratio of the real and imaginary parts of the energy. However, in graphene systems described by the Dirac equation, the spinor nature of the wave function and Klein tunneling rule out the possibility of any optical-like quasibound states. The discrete modes in the cavity, when the waveguide is absent or present, correspond to scattering resonances or Fano resonances, respectively. For a scattering resonance, its width is inversely proportional to the average lifetime of the quasiparticles in the cavity. For the $(l, m)=$ $(10,1)$ mode, in the absence of the waveguide, the scattering cross section exhibits a quite sharp peak (Appendix A), indicating a long lifetime and high quality confinement. As a result, when the waveguide is present, the corresponding
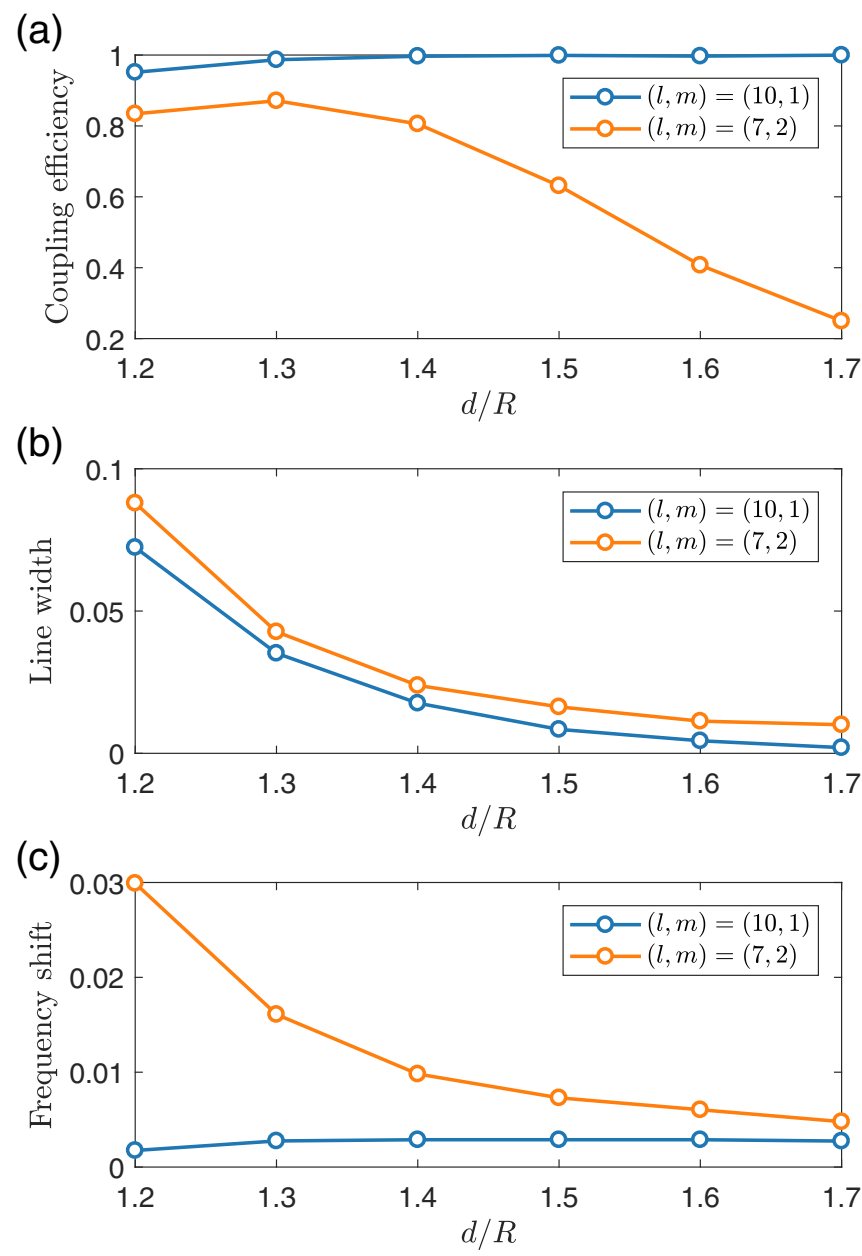

FIG. 4. Dependence of the characteristics of Fano resonance on the cavity-waveguide distance. [(a)-(c)] Coupling efficiency, resonance width, and frequency shift vs the normalized distance, respectively, for the two Fano resonances in Fig. 2.

Fano resonance is also sharp, which has little dependence on small variations in the distance between the cavity and the waveguide.

\section{Pseudospin polarization}

To further understand the emergence of the distance independent resonant modes, we examine the behavior of the pseudospin current in the waveguide and cavity at the critical coupling, which is calculated through the local expectation value of the Pauli matrices $\sigma$, where $\mathbf{j}=\langle\Psi|\sigma| \Psi\rangle$ is the pseudospin current density. The output pseudospin current in the waveguide can be obtained as the integration of the density as

$$
J_{x, y, z}(x)=\int_{-d-2 a}^{-d} j_{x, y, z}(x, y) d y .
$$

We impose the following normalization of the input current: $J_{x}(x=\infty)=1$, so a unity current magnitude corresponds to the case of perfectly pseudospin polarization. Figures 5(a) and 5(b) show both the in-plane and out-of-plane current distributions in the coupling region, for the resonant modes $(l, m)=(10,1)$ and $(l, m)=(7,2)$, respectively, where the 

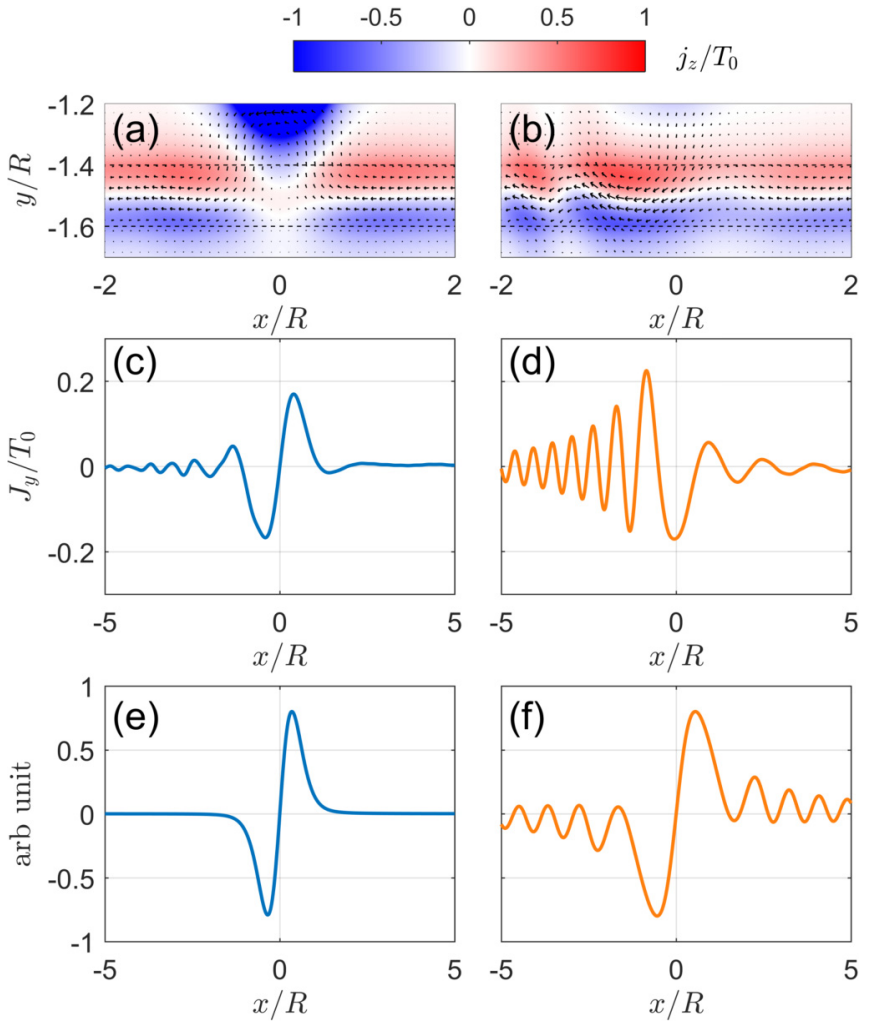

FIG. 5. Distinct behaviors of pseudospin currents associated with different types of resonant modes. (a) Current in the waveguide for the $(l, m)=(10,1)$ mode for critical coupling $k_{x} R=8.1906$. The black arrows represent the in-plane pseudospin current density given by the local expectation values of $\sigma_{x}$ and $\sigma_{y}$. The color represents the out-of-plane pseudospin current density given by the expectation value of $\sigma_{z}$. Associated with a resonance, the pseudospin current traveling directly from right to left dominates, in spite of the deviations near the waveguide and cavity boundaries in the coupling region. (b) Same picture for the resonant mode $(l, m)=(7,2)$, where both the in-plane and out-of-plane pseudospin currents exhibit large deviations from the propagation direction. [(c) and (d)] Integrated pseudospin currents over the $y$ direction for the $(l, m)=(10,1)$ and $(l, m)=(7,2)$ modes, respectively. (e) In the absence of the waveguide, pseudospin current profile near $y=-d$ for the scattering resonance corresponding to the $(l, m)=(10,1)$ mode, for which the quasiparticles have a long lifetime and the wave outside the cavity is dominantly outgoing. For this mode, the pseudospin polarization current is nonzero only near the cavity boundary. (f) A similar plot for the scattering resonance associated with the $(l, m)=(7,2)$ mode. The current in the $y$ direction exhibits an oscillation structure even at large distance from the coupling region.

input current from the right side is not pseudospin polarized. It can be seen that, for the $(l, m)=(10,1)$ mode [Fig. 5(a)], there are distortions in the current pattern near the cavity and waveguide boundaries in the coupling region but the current remains to be pseudospin unpolarized after passing through the region. In contrast, for the $(l, m)=(7,2)$ mode [Fig. 5(b)], the current structure is disturbed and exhibits oscillations even after passing through the cavity region and at a distance of a few cavity sizes. Further illustration of the distinct behaviors in the current can be seen by the integrated current (in the $y$ direction) as shown in Figs. 5(c) and $5(\mathrm{~d})$, with the normalization convention $J_{x}(x=\infty)=1$. Comparing with Fig. 5(c), the oscillations of the integrated pseudospin current even at large propagating distances in Fig. 5(d) are pronounced. Figures 5(e) and 5(f) show the current profiles for the corresponding scattering resonances when the waveguide is absent. The wave function associated with the mode $(l, m)=(10,1)$ is dominated by an outgoing wave described by the Hankel function of the first kind, but for the mode $(l, m)=(7,2)$, the wave function is a mixture of the Hankel functions of the first and second kind, signifying a scattering state. The results in Figs. 5(e) and 5(f) are similar to those in Figs. 5(a), 5(c) and 5(b), 5(d) respectively, indicating the role of cavity in inducing pseudospin polarization and the applicability of the mode coupling theory.

The phenomenon that the pseudospin current after passing the cavity becomes polarized is the result of the asymmetric potential applied. In fact, pseudospin polarization associated with a resonant mode depends on the properties of the corresponding scattering resonance. As shown in Fig. 5(e), when the scattering resonance has a long lifetime, outside the cavity the wave function is dominated by outgoing waves and the pseudospin polarized current is diminishingly small except near the coupling region. However, when the lifetime associated with a scattering resonance is short, a mixture of incoming and outgoing waves arises outside the cavity, making the pseudospin polarized current large, as shown in Fig. 5(f). Note that there is no relation between the quantum number $m$ and the type of scattering state. In general, if the potential height is much larger than the electron energy, scattering resonances with $m>1$ can exhibit a long lifetime.

\section{EXPLOITING DEFORMED CAVITY TO MODULATE PSEUDOSPIN}

As demonstrated in Sec. II, the presence of a cavity in the vicinity of a waveguide can have affect the pseudospin polarized current in the waveguide in a not-so-insignificant way. The effect can be quite dramatic when a Fano resonance emerges in the cavity. In particular, a beam of pseudospin unpolarized particles, after passing through the coupling region, can gain certain degree of pseudospin polarization. A specific example is presented in Figs. 5(c) and 5(d), where the integrated pseudospin polarization current along the $y$ direction is shown. The degree of polarization is position dependent and exhibits oscillations with a decaying amplitude away from the coupling region. The ability for a Fano resonance in the cavity to affect the pseudospin polarization of the quasiparticles in the waveguide suggests the possibility to use coupled cavitywaveguide system as a pseudospin modulator.

In spintronics applications $[6,8]$, it is desired to generate a strong, highly pseudospin polarized current. However, for a Fano resonance based pseudospin modulator, a trade off is inevitable: to achieve a high degree of pseudospin polarization, a strong coupling between the cavity and waveguide is required, but this leads to weak transmission through the waveguide. Does there exist a configuration that minimizes or even eliminates the trade off, i.e., is it possible to articulate a system that can generate both strong pseudospin polarization current and large transmission? Our answer is affirmative. 
Especially, in graphene systems, it has been demonstrated previously that geometrical deformation can enhance emission and tunneling [52]. Our idea is then to exploit a deformed cavity for pseudospin modulation.

For the waveguide-cavity coupled system, to generate geometrical deformation is experimentally feasible because all it takes is to modify the geometrical shape of the cavity from a perfect circle to, e.g., a stadium or the shape of Africa [40]. We note that, in optics, introducing appropriate geometric deformations to a microlasing cavity has the benefits of achieving both a high quality factor and strong directionality of emission [23]. In transport through a quantum dot or cavity scattering systems, utilizing a deformed cavity can also be advantageous [52,57,68,70,77-79].

To be concrete, we study a cavity with the shape of Africa $[39,40]$, which can be conveniently generated through a conformal mapping. In particular, defining $z=e^{i \theta}$ where $\theta \in$ $[0,2 \pi]$ for a circular cavity of unit radius in the $z$ plane, we obtain a deformed cavity in the $w$ plane through the following conformal mapping:

$$
w(z)=\frac{z+\delta_{1} z^{2}+\delta_{2} \exp \left(i \delta_{3}\right) z^{3}}{\sqrt{1+2 \delta_{1}^{2}+3 \delta_{2}^{2}}},
$$

where the geometry defined by the real and imaginary parts of $w$ has the same area as the original circle in the $z$ plane, and the choices of the parameters $\delta_{1}=\delta_{2}=0.2$ and $\delta_{3}=\pi / 3$ lead to a cavity of the shape of Africa. Note from Eq. (5) that the conformal mapping does not change the center of circle, so the distance between the deformed cavity and the waveguide can still be measured by $d$, the original distance between the circle and the waveguide.

We examine the transmission for the Africa cavity. For comparison, we also include the corresponding result for case of a circular cavity of the same area. The results are shown in Fig. 6, where the transmission $T_{0}$ versus the normalized input energy in the interval $k_{x} R \in[8,10]$ for the two cases is presented. For the circular cavity, in the energy interval displayed, there are four Fano resonances, as indicated by the four sharp and significant dips in the transmission curve. In contrast, for the deformed cavity, no such dip exists. The resonances can also be seen from the behavior of the total density of states (DOS) inside the cavity defined as

$$
\operatorname{DOS}=\iint_{\mathbf{r} \in \mathbb{D}_{\mathrm{II}}} \Psi^{\dagger}(\mathbf{r}) \Psi(\mathbf{r}) d \mathbf{r},
$$

where it exhibits four sharp peaks in the pertinent energy interval for the case of a circular cavity, each corresponding to a dip in the transmission, as shown in the inset of Fig. 6. The total DOS for the deformed cavity is nearly zero in the energy interval, except for a small and broad peak near the middle of the interval, indicating the emergence of only a weakly confined state at that energy. Apparently, chaos has removed all sharp Fano resonances, which also occurs with elastic scattering in graphene systems [68,77] and in the general context of quantum scattering (or transport) [52,57,68,70,7779].

While the results in Fig. 6 indicate that the presence of a deformed cavity near the waveguide has little effect on the transmission, is there any advantage to have such a cavity

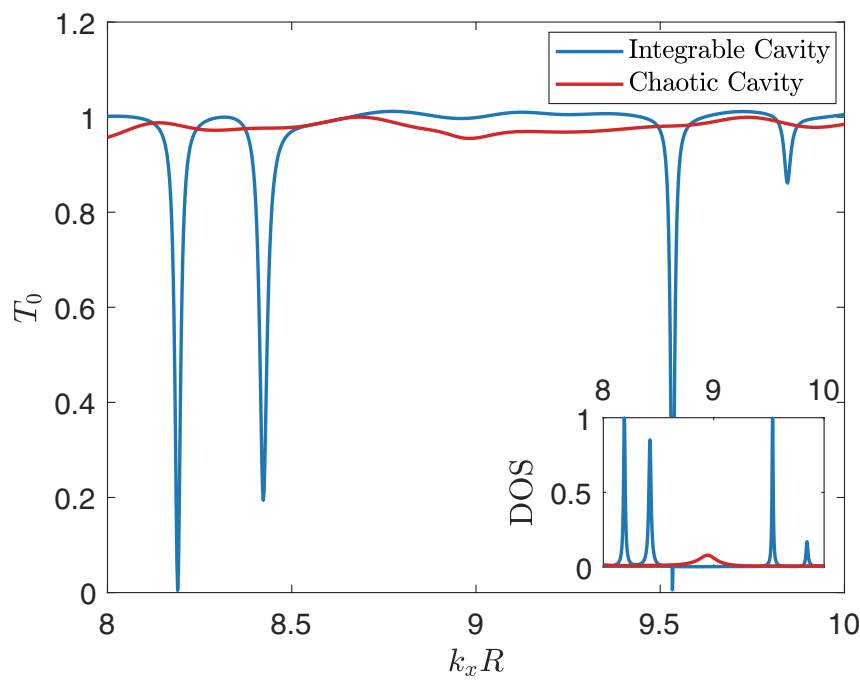

FIG. 6. Broadening of Fano resonances through geometrical deformation. Shown is the waveguide transmission vs the input normalized energy for the two cases where the coupled cavity is circular and has the shape of Africa, respectively. For the circular cavity (blue curve), four sharp Fano resonances arise, associated with which the transmission is significantly reduced. In contrast, for the deformed cavity, no such resonance exists and the value of the transmission maintains at near unity. These behaviors can also be seen from the plot of the total DOS inside the cavity vs the input energy, as shown in the inset, where a Fano resonance corresponds to a sharp peak in the total DOS.

from the point of view of pseudospin polarization? To answer this question, we examine how a particular resonant mode, e.g., the $(l, m)=(7,2)$ mode, is removed when the circular cavity is replaced by a deformed cavity.

We first study the case of a circular cavity. Figure 7(a) shows the pseudospin polarization current in the whole coupled system, where the length of the black arrows and the brightness of the color represent the relative magnitude of the pseudospin polarization current. Because of the resonant confinement in this case, the wave function is strongly localized in the cavity, giving rise to small transmission in the waveguide. Figure 7(b) shows the combined pseudospin polarization current in the $(y, z)$ plane in the propagation direction (thick green arrow), where the negative $J_{x}$ component is not included here for clarity. In the absence of the cavity, we have $J_{y}=J_{z}=0$ due to the symmetry of the applied potential that defines the waveguide. The interaction with the cavity causes the pseudospin polarization current to possess a component in the $(y, z)$ plane, and the current persists even at some distance passing through the coupling region, which can be better seen from the plot of $J_{z}$ versus $x$, as shown in Fig. 7(c). This behavior resembles that of electron scattering from a magnetic domain wall [80].

We next investigate the case of a deformed cavity of the Africa shape. Figure 7(d) shows the behavior of the pseudospin polarization current through the coupling region. For a meaningful comparison with the case of the circular cavity, we use the same input energy as that in Figs. 7(a)-7(c). Due to the weak coupling and removal of the Fano resonance by chaos, the current concentrates in the waveguide. The weak 

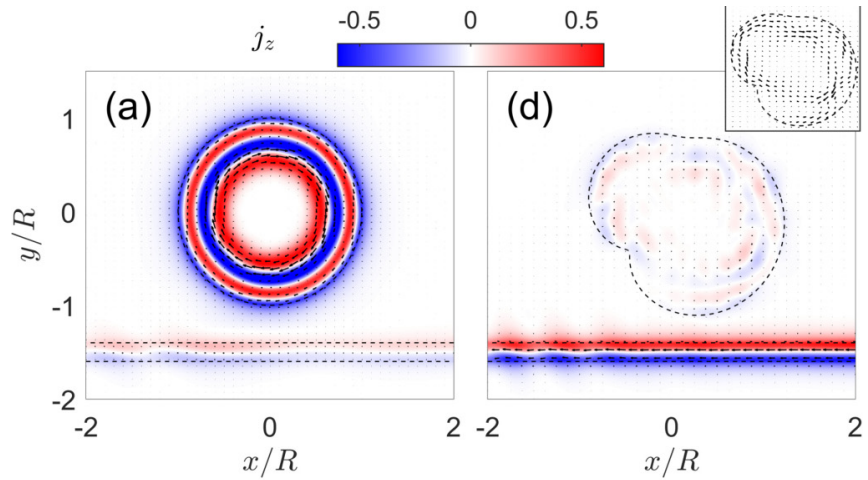

(b)

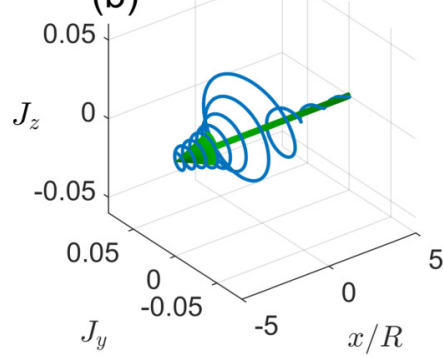

(e)
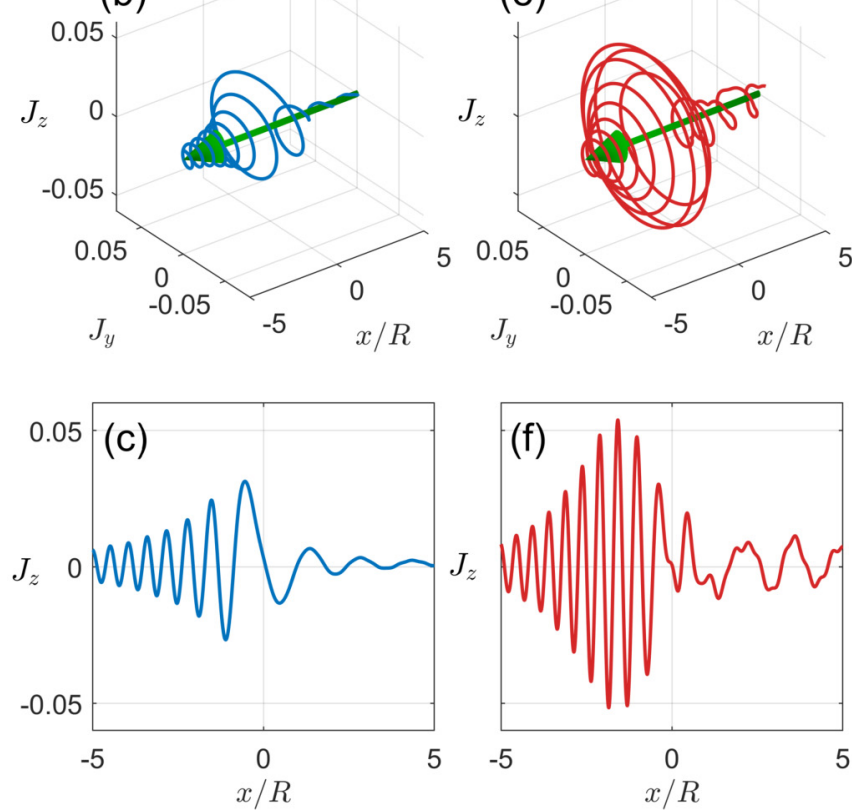

FIG. 7. Direct evidence of chaos assisted tunneling and pseudospin polarization enhancement. (a) For the case of a circular cavity, for $k_{x} R=8.4231$ so that the $(l, m)=(7,2)$ resonant mode is excited, distribution of the pseudospin polarization current density in the whole coupled system. The black arrows indicate the in-plane current and the color represents the out-of-plane current $j_{z}$. The direction of the in-plane current in the waveguide is dominated by the input direction (from right to left), see Fig. 5(d). (b) The current components $J_{y}$ and $J_{z}$ along the waveguide, where the thick green arrow indicates the direction of the input spinor wave. (c) Integrated out-of-plane pseudospin polarization current $J_{z}$ vs $x$, which exhibits oscillations even away from the coupling region at distances of several times of the cavity size. [(d)-(f)] Results corresponding to those in (a)-(c) but for a deformed cavity of the same area for $k_{x} R=$ 8.9623. In (d), the DOS inside the cavity is much less pronounced than that in (a), with the inset depicting the in-plane current. [(e) and (f)] A relatively strong pseudospin polarization current.

current inside the cavity, however, is clockwise, indicating that only modes with $l>0$ in the cavity are activated through the coupling with the waveguide. Figures 7(e) and 7(f) show, respectively, the pseudospin polarization current in the $(y, z)$ plane and the $z$ component versus $x$. Comparing with the corresponding results for the case of circular cavity [Figs. 7(b) and $7(\mathrm{c})]$, we wee that the magnitude of the pseudospin polarization current is almost doubled. Thus not only is geometrical deformation able to suppress the Fano resonance and significantly enhances transmission through the waveguide, but it also has the benefit of producing stronger pseudospin polarization.

Another feature of geometrical deformation enhanced pseudospin polarization is that, in comparison with the case of a circular cavity, the oscillation frequency of the pseudospin polarization current is larger, as can be seen from Fig. 7(f). From the point of view of mode coupling, the oscillation frequency is determined by the scattered radial wave. For a deformed cavity, waves associated with different angular momentum quantum numbers are effectively mixed, giving rise to a wider scattering profile in comparison with the case of a circular cavity where only a single mode is instigated for a given input energy value [81]. In general, for a circular or a deformed cavity, a large number of resonances can arise for different amount of coupling, each generating a certain degree of pseudospin polarization.

For spin modulation in graphene, there were experimental characterizations for gate controlled devices in terms of the spin injection efficiency (or fidelity) and the spin diffusion length $[6,8]$, where the former measures the percentage of the spin polarized current and the latter quantifies the effective spatial region in which the spin polarized current can sustain. The value of fidelity [82] can be larger than $60 \%$ and the diffusion length can be several $\mu \mathrm{m}$ [83]. Motivated by the experimental results on characterization of the real spin polarization, we define the pseudospin fidelity for our coupled cavity-waveguide system in terms of the $y$ and $z$ components of the pseudospin polarization vector as

$$
F=\frac{\sqrt{J_{y}^{2}+J_{z}^{2}}}{\left|J_{x}\right|},
$$

which depends on the input energy and the distance between the cavity and the waveguide. Intuitively, in the weak coupling regime, the value of $F$ is small, as the modes in the cavity do not have a significant impact on the pseudospin polarization current in the waveguide. Associated with a Fano resonance, the fidelity value can be (locally) maximized. Away from the coupling region along the waveguide, the fidelity should exhibit an oscillatory behavior with an decreasing amplitude, as implied by the results in Figs. 7(b) and 7(e). Along the waveguide, at locations near the coupling region, on average the fidelity value is large, facilitating experimental observation.

When calculating the fidelity, a technical difficulty is that the numerically required truncation of the waveguide in the propagation direction can make the transmission in the absence of any cavity deviate from unity (Appendix B). If the distance $d$ between the waveguide and cavity is small, the cavity effectively serves as a barrier, generating large errors in the calculation of the transmission and reflection coefficients. However, when $d$ is reasonably large, the fidelity value is not affected by the finite truncation (Appendix D). Figure 8(a) shows, at a fixed location, the $F$ value versus the input energy for the circular and deformed cavities. We see that $F$ reaches a local maximum when a Fano resonance arises. For the integrable cavity, this occurs for the $(l, m)=(7,2)$ 

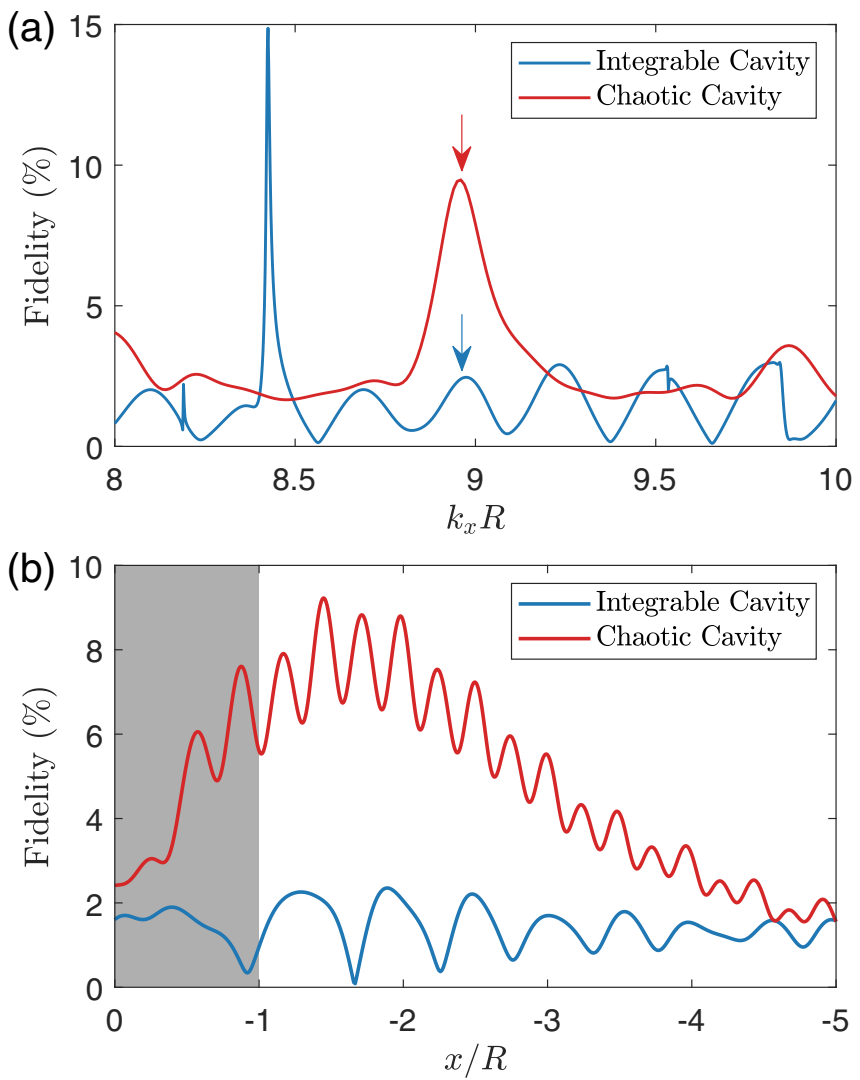

FIG. 8. Fidelity and diffusion length characterizing pseudospin polarization. (a) For the circular cavity (blue trace), fidelity for $k_{x} R \in$ $[8,10]$ measured at $x=-2 R$. Associated with a Fano resonance, the fidelity reaches maximum. The red curve is the fidelity plot for the deformed cavity, where a local maximum also arises. (b) Fidelity vs distance for the input energy value indicated by the downward arrows in (a). The gray region illustrates the cavity size. For the deformed cavity, the fidelity maintains at some large value even at distances of several cavity sizes.

mode. For the deformed cavity, the maximum $F$ value occurs for $k_{x} R \approx 8.95$. Figure 8 (b) shows, for this value of $k_{x} R$, $F$ versus the testing location along the waveguide, which exhibits an oscillatory behavior with a decreasing magnitude on average. Even at location several times of the cavity size away from the coupling region, the fidelity value is still quite appreciable, indicating a similar value for the pseudospin diffusion length and rendering feasible an experimental study of our coupled cavity-waveguide system as a pseudospin modulator.

\section{NULL PSEUDOSPIN POLARIZATION IN A SYMMETRICALLY COUPLED CAVITY-WAVEGUIDE CONFIGURATION}

Pseudospin polarization arises from the asymmetry in the applied electrical potential, i.e., a waveguide side-coupled with a cavity. It is useful to consider the symmetrical coupled system of two identical cavities coupled to the waveguide, one on each side. The expectation is that, because of the symmetry, pseudospin polarization vanishes.
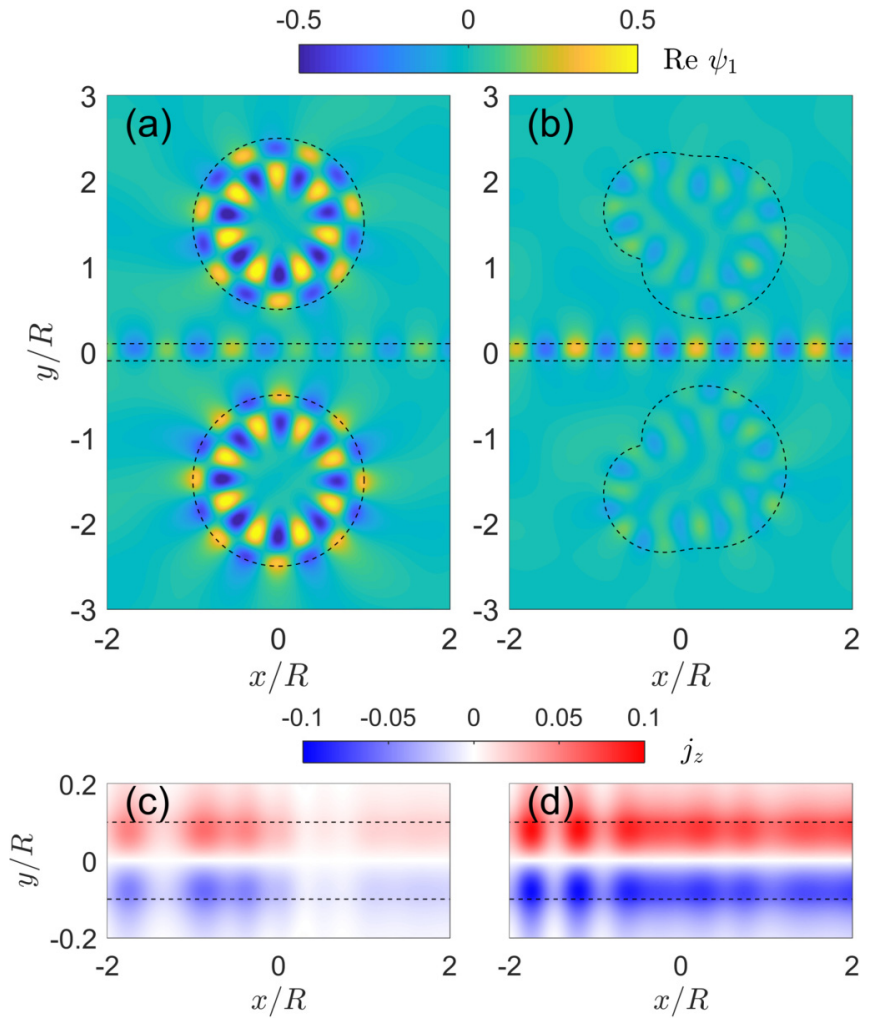

FIG. 9. Disappearance of pseudospin polarization current in a symmetrically coupled cavity-waveguide system. (a) Distribution of the real part of the first spinor component for a symmetric system of two circular cavities, one on each side of the waveguide. The input spinor wave is the same as that in Fig. 7(a). The nodal numbers in the upper and lower cavities are different, due to the degeneracy of the modes $(l, m)=(7,2)$ and $(l, m)=(-8,2)$, as reflected by the sign of $\phi$ in Eq. (3). (b) The corresponding wave-function distribution for the deformed cavity of the Africa shape. [(c) and (d)] Distributions of the $z$ component of the pseudospin polarization current density $j_{z}$ in the waveguide for the integrable and chaotic cavity, respectively. The density distribution is perfectly antisymmetric with respect to the central axis of the waveguide, giving rise to $J_{z}=0$.

We generalize the MMP method to calculate the wave functions and the pseudospin polarization current for the symmetrically coupled system by placing two additional sets of poles: one inside and another outside of the second cavity domain (Appendix C). The procedure to construct the wave function is similar to the case of asymmetrically coupled system, where the wave function outside the two cavities and waveguide is determined by all the poles inside those domains. The poles outside but near each domain gives the wave function inside that domain.

Figure 9 shows the results on the pseudospin polarization current of the symmetrically coupled cavity-waveguide system. In particular, Fig. 9(a) shows the real part of $\psi_{1}$ for the circular cavity associated with a Fano resonance. Note that the numbers of nodes in the upper and lower cavities are different, due to the opposite signs of the angular momentum in the cavities. The corresponding result for the case of deformed Africa cavity is shown in Fig. 9(b). Figures 9(c) and 9(d) show the pseudospin polarization current density inside the waveguide for the circular and deformed cavities, respectively. 
Comparing with the results of the asymmetrically coupled system in Figs. 7(a) and 7(d), we see that the pseudospin polarization current density is now perfectly asymmetrically distributed in the space with respect to the central axis of the waveguide, regardless of whether the cavity is circular or deformed. This leads to $J_{z}=0$.

\section{DISCUSSION}

To effectively manipulate pseudospin polarization is key to spintronics. We have articulated a device configuration and studied scenarios to generate and enhance pseudospin polarization in graphene. Especially, for propagation through a graphene waveguide, placing a cavity nearby can generate certain degree of pseudospin polarization for initially unpolarized quasiparticles. For a circular cavity, sharp Fano resonances can arise. For some specific incident energy that induces a Fano resonance, a high degree of pseudospin polarization can be achieved. However, the energy interval to achieve such an enhancement is quite narrow because of the sharpness of the Fano resonance.

There are thus two difficulties to exploit Fano resonances in a circular cavity to realize strong pseudospin polarization. Firstly, the resonances are typically sharp, making tuning to it practically difficult. Secondly, associated with a Fano resonance, transmission through the waveguide can be significantly reduced. It is infeasible to achieve high transmission and a sizable pseudospin polarization at the same time. Our central idea to overcome the difficulties is to exploit geometrical deformation. In this case, Fano resonances are broadened. The remarkable phenomenon is that a deformed cavity can enhance pseudospin polarization in a wide energy interval and is thus more experimentally feasible. Because of the weakening of the Fano resonance, high transmission can be achieved simultaneously. Using a deformed cavity to generate and modulate pseudospin polarization is thus desired spintronics device applications.

We discuss a few pertinent issues. (1) Effect of simultaneous presence of two valleys on pseudospin polarization. In our cavity-waveguide coupled system, there is no inversion symmetry about the $y$ axis (note that $x$ is the propagation axis in the waveguide). The result that pseudospin unpolarized incoming electrons from the right gain certain degree of pseudospin polarization after passing through the coupling region was obtained for one valley, as demonstrated in Figs. 7(a) and 7(d), where the asymmetric wave function makes the pseudospin polarized. The presence of the other nonequivalent valley can lead to cancellation of pseudospin polarization in some directions, but not in all directions, depending on the orientation of the lattice structure. For example, say the Hamiltonian for one valley is written as $H_{K}=v_{g}\left(\sigma_{x} p_{x}+\sigma_{y} p_{y}\right)$. If the orientation in the $x$ direction is of the armchair type, the Hamiltonian associated with the other valley $(K)$ is [3] $H_{K^{\prime}}=v_{g}\left(-\sigma_{x} p_{x}+\sigma_{y} p_{y}\right)$. If the spinor wave function for the $K$ valley is written as $\psi=\left[\psi_{A}, \psi_{B}\right]^{T}$, then the solution for the $K^{\prime}$ valley is $\psi=\left[\psi_{B},-\psi_{A}\right]^{T}$. The relations between the pseudospin current components of the two valleys are $j_{x}=-j_{x}^{\prime}, j_{y}=j_{y}^{\prime}$ and $j_{z}=-j_{z}^{\prime}$. That is, the $y$ component of pseudospin polarization associated with $K^{\prime}$ has the same sign as that with the $K$ valley, but the signs of the
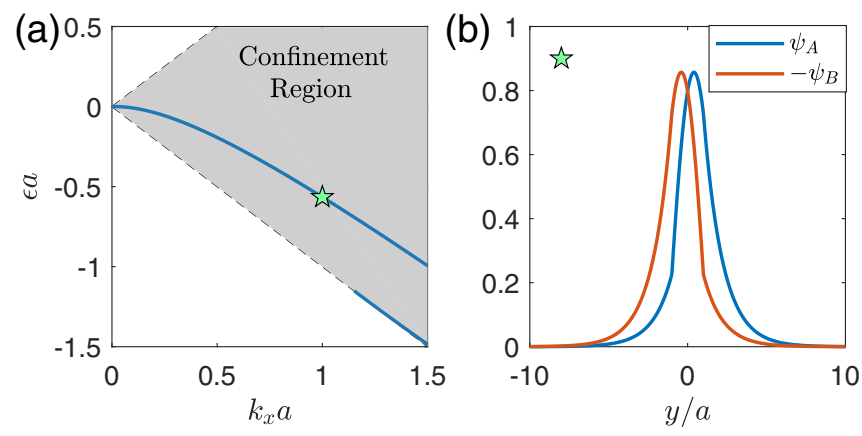

FIG. 10. An illustrative example of confinement conditions and wave function in a graphene waveguide in the single mode operation regime. (a) Dispersion relationship for a graphene waveguide with $V_{3} a=0.8$. For $k_{x}$ small, only a single mode exists. The shaded region is associated with the confinement condition $|\epsilon|<\left|k_{x}\right|$. The green pentagram denotes $k_{x} a=1$. (b) Wave function for $k_{x} a=1$.

pseudospin polarization in the $x$ and $z$ directions associated with the two valleys are opposite. There is thus a time reversal symmetry: pseudospin unpolarized current associated with valley $K$ coming from the right to the left becomes polarized after passing through the coupling region, and pseudospin polarized current associated with $K^{\prime}$ coming from the left will emerge pseudospin unpolarized on the right. Overall, in the direction from right to left, coupling with the cavity can still generate pseudospin polarization in the $y$ direction.

(2) Experimental detection of pseudospin. Experimentally, directly detecting the pseudospin can be challenging [7], but it is not infeasible. In particular, the methods of sublattice resolved LDOS mapping [9] and polarization resolved photoluminescence spectroscopy [84] have been used to investigate the sublattice pseudospin dynamics, based on the physical principle that the sublattice pseudospin alignment/texture will affect the carrier optical properties. We are thus hopeful that the results in this paper can be tested experimentally.

(3) Multimode operation of waveguide. Multimode graphene waveguides have been previously studied $[28,29]$, which arises if $k_{x}$ is sufficiently large. The conductance is proportional to the number of modes. The dispersion relationship shown in Fig. 10 stipulates that the energies associated with different modes are different. This means that, if one mode in the waveguide is already coupled to the cavity at some value of $k_{x}$, then the coupling between other modes and the cavity must necessarily be much weaker and can be ignored. That is the reason that we focus on the single mode case. However, a guiding mode can couple to multiple modes in the cavity, which occurs when the confinement of the cavity is sufficiently strong so that different scattering resonances are simultaneously present inside the cavity. A similar phenomenon has been observed in optics, which leads to an asymmetric transmission profile [73]. In this case, pseudospin polarization is mainly determined by coupling with the mode associated with the strongest confinement.

(4) Development of a semiclassical theory. Classically, a deformed cavity generates chaos. In the study of quantum chaos, semiclassical theory has played an important role [51,85-92]. The question is whether a semiclassical theory 
can be developed to understand pseudospin modulation in the coupled cavity-waveguide system.

In the semiclassical regime, the Planck constant is infinitesimal: $\hbar \rightarrow 0$. In this regime, there is a correspondence between the quantum and classical statistical properties of the system. There are three difficulties in developing a semiclassical theory for the coupled cavity-waveguide system.

The first difficulty is to develop a Green's function formalism. This can be done when the system configuration is of the quantum-dot type [93,94]: a cavity plus two leads, where semiclassical transport can be studied by the standard Green's function method. Even though the leads are semi-infinite, the whole quantum dot system can be regarded as a single entity. Our system involves three regions: the infinite waveguide that cannot be treated as a lead, the finite cavity, and the semiinfinite space between the cavity and waveguide. The input wave function propagates in the waveguide. When it arrives at the coupling region, it interacts with the cavity through evanescent waves, induces resonances in the cavity, and scatters back into the waveguide. This process continues until an equilibrium is reached. While this kind of configurations has been studied recently in optics [73], to calculate the total wave function for the coupling system, whether a Green's function formalism can be developed and whether the formalism, if feasible, can be extended to Dirac spinor systems remain to be open questions.

The second difficulty lies in calculating the averages over the energy, as is required for establishing a correspondence between quantum and classical calculations, e.g., level spacing statistics in a closed system and the average conductance through a quantum dot. The physical reason is that the energy is always specified within a small interval $\Delta E$ that is classically small but quantum mechanically large (in comparison with the average level spacing). For our two-body coupled system, it is necessary to take the energy average for both the cavity and waveguide. It is not clear how this average can be done in a computationally feasible manner, especially in the case where the waveguide permits multiple modes of propagation.

The third difficulty is the determination of the classical periodic orbits. For a single closed cavity, classical periodic orbits can be defined straightforwardly. For a quantum dot system, this can also be done for the dot region. In our case, the cavity is defined by some electrical potential. At the boundary of the cavity, reflection and transmission through the mechanism of Klein tunneling can occur. An optical analogy is light traveling a medium with different refractive indices [95]. It is not clear at the present how closed classical orbits can be defined in our system where scattering occurs in the two-dimensional semi-infinite region between the cavity and the waveguide.

\section{ACKNOWLEDGMENT}

We would like to acknowledge support from the Vannevar Bush Faculty Fellowship program sponsored by the Basic Research Office of the Assistant Secretary of Defense for Research and Engineering and funded by the Office of Naval Research through Grant No. N00014-16-1-2828.

\section{APPENDIX A: SCATTERING SOLUTIONS OF A CAVITY IN GRAPHENE}

To solve the transport/scattering problem with the coupled cavity-waveguide system, it is necessary to obtain the solutions of the cavity and waveguide separately. Consider a single cavity in a graphene sheet generated by the following electrical potential:

$$
U_{\mathrm{II}}(x, y)= \begin{cases}v_{g} \hbar V_{2}, & r<R, \\ 0, & r>R,\end{cases}
$$

with the corresponding wave vectors $k_{2}=\left|\epsilon-V_{2}\right|$ and $k_{1}=$ $|\epsilon|$, respectively. The band indices inside and outside the cavity are $\tau_{2}=\operatorname{sign}\left(\epsilon-V_{2}\right)$ and $\tau_{1}=\operatorname{sign}(\epsilon)$, respectively. For our coupled cavity-waveguide system, the resonant states in the cavity are of particular importance. We use three different methods to calculate and compare the cavity resonant states.

\section{Cavity resonant states generated by a plane wave in the} setting of elastic scattering

Let the incident wave be

$$
\chi^{\text {in }}=\exp (i \in x)\left(\begin{array}{l}
1 \\
i
\end{array}\right) .
$$

Using the Jacobi-Anger identity [96]

$$
e^{i x} \equiv \sum_{l=-\infty}^{\infty} i^{l} J_{l}(r) e^{i l \theta},
$$

we expand the plane wave into spherical waves:

$$
\chi^{\text {in }}=\exp (i \in x)\left(\begin{array}{l}
1 \\
i
\end{array}\right)=\sum_{l} i^{l}\left(\begin{array}{c}
J_{l}\left(k_{1} r\right) \\
i \tau_{1} J_{l+1}\left(k_{1} r\right)
\end{array}\right) e^{i l \theta} \text {, for } r>R \text {. }
$$

The wave functions outside the cavity can be written in terms of the Bessel functions as

$$
\Psi^{(\mathrm{I})}=\sum_{l} i^{l} g_{l}\left(\begin{array}{c}
H_{l}^{(1)}\left(k_{1} r\right) \\
i \tau_{1} H_{l+1}^{(1)}\left(k_{1} r\right)
\end{array}\right) e^{i l \theta}, \text { for } r>R .
$$

Similarly, the wave function inside the cavity can be expressed as

$$
\Psi^{(\mathrm{II})}=\sum_{l} i^{l} p_{l}\left(\begin{array}{c}
J_{l}\left(k_{2} r\right) \\
i \tau_{2} J_{l+1}\left(k_{2} r\right)
\end{array}\right) e^{i l \theta}, \text { for } r<R .
$$

Matching the boundary condition at $r=R$ :

$$
\left(\chi^{\text {in }}+\Psi^{(\mathrm{I})}\right)_{r=R}=\left(\Psi^{(\mathrm{II})}\right)_{r=R},
$$

we get

$$
\begin{aligned}
J_{l}\left(k_{1} R\right)+g_{l} H_{l}^{(1)}\left(k_{1} R\right) & =p_{l} J_{1}\left(k_{2} R\right), \\
J_{l+1}\left(k_{1} R\right)+g_{l} H_{l+1}^{(1)}\left(k_{1} R\right) & =\tau_{1} \tau_{2} p_{l} J_{l+1}\left(k_{2} R\right),
\end{aligned}
$$

with the solutions:

$$
\begin{aligned}
g_{l} & =-\frac{J_{l}\left(k_{2} R\right) J_{l+1}\left(k_{1} R\right)-\tau_{1} \tau_{2} J_{l}\left(k_{1} R\right) J_{l+1}\left(k_{2} R\right)}{J_{l}\left(k_{2} R\right) H_{l+1}^{(1)}\left(k_{1} R\right)-\tau_{1} \tau_{2} J_{l+1}\left(k_{2} R\right) H_{l}^{(1)}\left(k_{1} R\right)}, \\
p_{l} & =\frac{J_{l}\left(k_{1} R\right) H_{l+1}^{(1)}\left(k_{1} R\right)-H_{l}^{(1)}\left(k_{1} R\right) J_{l+1}\left(k_{1} R\right)}{J_{l}\left(k_{2} R\right) H_{l+1}^{(1)}\left(k_{1} R\right)-\tau_{1} \tau_{2} J_{l+1}\left(k_{2} R\right) H_{l}^{(1)}\left(k_{1} R\right)} .
\end{aligned}
$$


The circular symmetry stipulates $g_{l}=g_{-l-1}$ and $p_{l}=$ $\tau_{1} \tau_{2} p_{-l-1}$, i.e., every resonance contains equal contributions from the $l$ th and $-(l+1)$ th states. Note that $g_{l}$ and $p_{l}$ share the same denominator that can be zero, corresponding to an infinitely sharp resonance. For these states, the outside the cavity is dominated by outgoing waves.

\section{Cavity resonant states generated by evanescent waves}

Because the modes in the cavity and in the waveguide are coupled through evanescent waves, only angular momentum with $l>0$ will appear. This is different from plane-wave scattering where a resonance couples the states with angular momentum $l$ and $-l-1$ on the equal footing. See Sec. II for details, where the expansion in terms of the evanescent waves is given by Eq. (3).

\section{Cavity resonant states generated through an optical approach}

Recall that, in optics, the quality factor of the cavity is given by the real and imaginary parts of the energy as $Q=$ $-\operatorname{Re}(\omega) / 2 \operatorname{Im}(\omega)$, where $\omega=c k$ and each mode corresponds to a scattering resonance (a quasibound state) [23,76]. In Dirac systems, such resonant states can be generated by imposing outgoing waves only outside the cavity. Specifically, the wave functions outside and inside the cavity can be written as

$$
\Psi^{(\mathrm{I})}(r, \theta)=a_{l}\left(\begin{array}{c}
H_{l}^{(1)}\left(k_{2} r\right) \\
i H_{l+1}^{(1)}\left(k_{2} r\right) e^{i \theta}
\end{array}\right) e^{i l \theta}, \text { for } r>R,
$$

and

$$
\Psi^{(\mathrm{II})}(r, \theta)=b_{l}\left(\begin{array}{c}
J_{l}\left(k_{1} r\right) \\
i J_{l+1}\left(k_{1} r\right) e^{i \theta}
\end{array}\right) e^{i l \theta}, \text { for } r<R,
$$

respectively, where $a_{l}$ and $b_{l}$ are coefficients that can be determined by the boundary conditions and normalization. Let

$$
C_{l}=\frac{J_{l}\left(k_{1} R\right)}{J_{l+1}\left(k_{1} R\right)} \text { and } D_{l}=\frac{H_{l}^{(1)}\left(k_{2} R\right)}{H_{l+1}^{(1)}\left(k_{2} R\right)} .
$$

The continuity of the wave function at the boundary is guaranteed when, for a given angular momentum quantum number $l$, the following holds:

$$
F_{l} \equiv \frac{\left|C_{l}-D_{l}\right|}{\left|C_{l}\right|+\left|D_{l}\right|}=0,
$$

where the denominator is the normalization factor.

There are two major differences between resonant states in optics and in Dirac systems. Firstly, for a microcavity in optics $[23,76]$, the resonances correspond to solutions in $k \in \mathbb{C}$. For an imaginary value of $k$, due to the dispersion $\omega=c k$, the energy is also imaginary, which physically means loss of energy in time. The quality factor characterizing the confinement is thus given by $Q=-\operatorname{Re}(\omega) / 2 \operatorname{Im}(\omega)$. However, in Dirac systems, some states can appear for $k \in \mathbb{R}$, which corresponds to a real energy and does not decay with time. Secondly, in optics, associated with each resonant state, there is a peak in the scattering cross section with its width inversely proportional to the lifetime. In a Dirac scattering system, $F_{l}$ has a minimum value that is not zero. Quasibound states similar to those in optics are thus not well defined in Dirac systems.
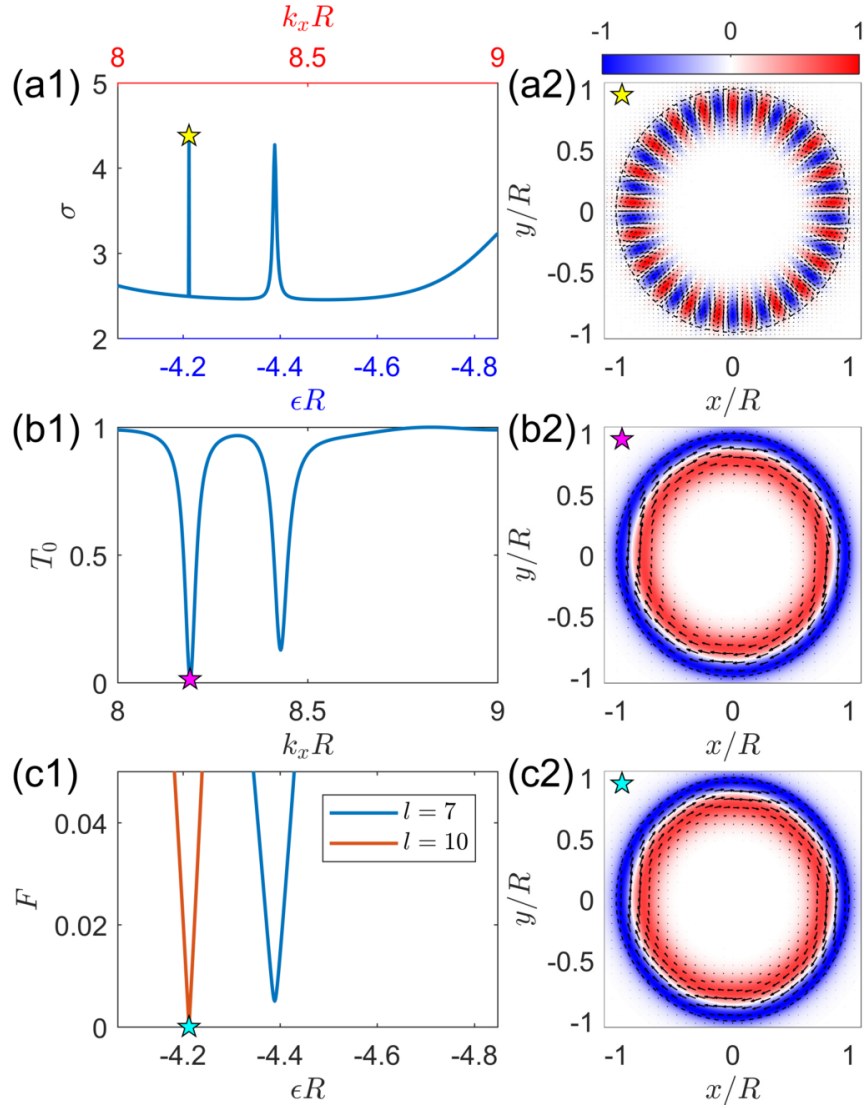

FIG. 11. Comparison of representative graphene cavity resonant states obtained from three methods: [(a1) and (a2)] elastic plane wave scattering, [(b1) and (b2)] evanescent waves, and [(c1) and (c2)] optical analogy. The normalized potential is $V_{2} R=8$. (a1) Scattering cross section vs the incident energy (or the wave vector $k_{x}$ ), where the yellow pentagram specifies a sharp resonance mode. (a2) Wave -function profile associated with the resonance mode at $\epsilon R=-4.2125$, where the black arrows indicate the in-plane current and the color represents the local expectation value of $\sigma_{z}$. As the scattering process involves simultaneously the angular momentum modes $l$ and $-l-1$, there is no net current. (b1) Transmission vs the input energy, where the system is identical to that in Fig. 5(a) and the pink pentagram indicates a Fano resonance mode. (b2) The wave-function profile of the Fano resonance mode in (b1), where the arrow and color legends are the same as those in (a2). A clockwise current appears. (c1) The quantity $F$ in Eq. (A13) vs energy. A solution for $l=10$ exists but not for $l=7$, indicating that a quasibound mode with outgoing wave only can emerge for $l=10$. The blue pentagram represents one quasibound state solution. (c2) The corresponding wave-function profile for the quasibound mode for $(l, m)=(10,1)$.

\section{Comparison of cavity resonant states obtained from the three methods}

Figure 11 shows some representative resonant modes obtained from the three methods, where the top (panels a1 and a2), middle (panels b1 and b2), and bottom (panels c1 and c2) rows show two resonant states generated by the elastic plane wave scattering, evanescent waves, and the optical method, respectively. As shown in Figs. 11(a1), 11(b1), and 11(c1), the modes emerge at the same energy (or wave vector $k_{x}$ ) value. 
The profile of the corresponding wave function indicates that the elastic scattering state has local current only (a2), but the Fano resonance generated by evanescent wave coupling (b2) and the optical method (c2) have a biased global current.

For another resonant mode with $(l, m)=(7,2), F_{l}$ is small but not zero, indicating that a quasibound solution with outgoing wave only does not exist. However, there is still a scattering resonance.

\section{APPENDIX B: GRAPHENE WAVEGUIDE}

We calculate the wave-function solutions for a graphene waveguide in the absence of cavity. The electrical potential that defines a waveguide is

$$
U_{\mathrm{III}}(x, y)= \begin{cases}v_{g} \hbar V_{3}, & |y| \leqslant a, \\ 0, & |y|>a,\end{cases}
$$

where the traveling mode is along the $x$ direction and confinement occurs in the $y$ direction with $y=0$ being the central axis. The eigenequation is

$$
H\left(\begin{array}{l}
\psi_{1}(y) \\
\psi_{2}(y)
\end{array}\right) e^{i k_{x} x}=E e^{i k_{x} x}\left(\begin{array}{l}
\psi_{1}(y) \\
\psi_{2}(y)
\end{array}\right) .
$$

Introducing $E \equiv v_{g} \hbar \epsilon$, we have

$$
\begin{aligned}
& \left(V_{3}-\epsilon\right) \psi_{1}+\left(k_{x}-\frac{d}{d y}\right) \psi_{2}=0, \\
& \left(k_{x}+\frac{d}{d y}\right) \psi_{1}+\left(V_{3}-\epsilon\right) \psi_{2}=0 .
\end{aligned}
$$

Letting $\psi_{A} \equiv \psi_{1}-\psi_{2}$ and $\psi_{B} \equiv \psi_{1}+\psi_{2}$, we get

$$
\begin{aligned}
& \left(V_{3}-\epsilon+k_{x}\right) \psi_{B}+\frac{d}{d y} \psi_{A}=0, \\
& \left(V_{3}-\epsilon-k_{x}\right) \psi_{A}-\frac{d}{d y} \psi_{B}=0 .
\end{aligned}
$$

Since $V_{3}$ is a constant except at the boundary, we can decouple Eq. (B4) as

$$
\frac{d^{2}}{d y^{2}} \psi_{A}+\left[\left(V_{3}-\epsilon\right)^{2}-k_{x}^{2}\right] \psi_{A}=0 .
$$

The solution for $\psi_{A}$ is given by

$$
\psi_{A}= \begin{cases}\alpha e^{i \alpha_{1} y}+\beta e^{-i \alpha_{1} y}, & |y| \geqslant a \\ \gamma e^{-\alpha_{2} y}, & y>a \\ \eta e^{\alpha_{2} y}, & y<-a\end{cases}
$$

where $\alpha_{1} \equiv \sqrt{\left(V_{3}-\epsilon\right)^{2}-k_{x}^{2}}$ and $\alpha_{2} \equiv \sqrt{k_{x}^{2}-\epsilon^{2}}$. For a confinement mode, $\alpha_{2}$ must be a real positive number. The confinement region can then be defined as $|\epsilon|<\left|k_{x}\right|$. For $\psi_{A}$ with an even (odd) symmetry, we have $\alpha=\beta(\alpha=-\beta)$.

The boundary condition for the Dirac equation is that the two spinors must be continuous at the boundary, stipulating that $\psi_{A}$ and $\psi_{B}$ must be continuous at $y= \pm a$. We can then (a)

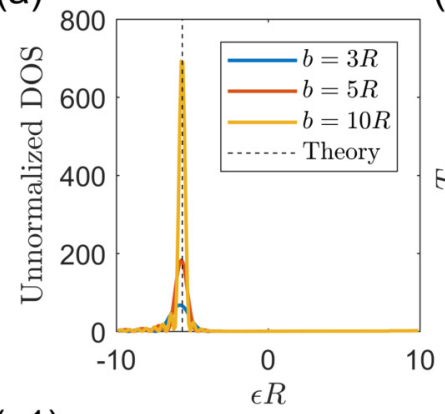

(b)

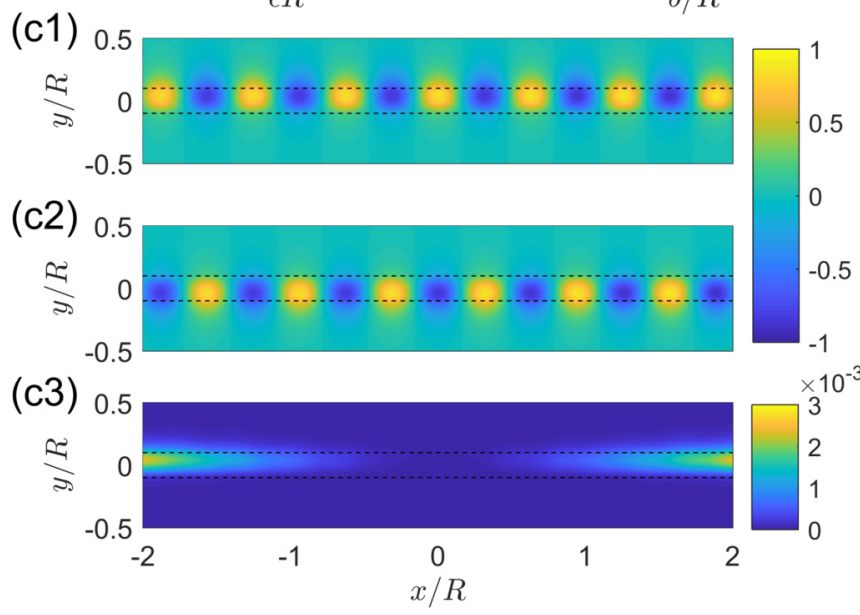

FIG. 12. Test and validation of the MMP method for an isolated graphene waveguide. For convenience, the width of the waveguide is chosen to be $a=R / 10$, where $R$ is the radius of the circular cavity in the coupled system. (a) For given $k_{x} R=10$, a bound state exists for $\epsilon \in\left(-k_{x}, k_{x}\right)$. The free-space average DOS, as defined in Eq. (C6), exhibits a peak for $\epsilon R \approx-7$, regardless of the truncation length of the waveguide insofar as it is much larger than the width. (b) Free-space transmission $T_{0}=J_{x}(x=-R) / J_{x}(x=$ $R)$ vs the relative truncation length. [(c1) and (c2)] Real part of $\psi_{1}$ and $\psi_{2}$, respectively, calculated from the MMP method. (c3) The relative error $\delta \Psi=\left\|\Psi-\Psi_{0}\right\|$, which is exceedingly small, attesting to the applicability of the MMP method to the graphene waveguide.

determine the coefficients in Eq. (B6). An example is shown in Fig. 10, which illustrates that, for small values of $k_{x}$, the waveguide is in single mode operation. For $V_{3} a=0.8$, this occurs for $k_{x} a<1.1$. Our central task is to treat the coupled cavity-waveguide system, for which no analytic method is available to calculate the wave function. As discussed in the main text, a viable approach is the MMP method [62-66] adopted to relativistic quantum spinor systems [68-70]. To test the applicability of the MMP method as applied to the coupled system, it is useful to examine its ability to calculate the wave function of the single traveling mode inside the waveguide that decays exponentially in the $y$ direction. To do this, we truncate the waveguide to a finite length $2 b$, which is much larger than its width: $b \gg a$. An example of the eigenstate is shown in Fig. 12(a). For an appropriate value $\epsilon$ of the input energy, a resonance can arise, the peak of which increases with $b$. Figure 12(b) shows the transmission versus the truncation length in the free space. It can be seen that, as the length of the waveguide increases, 
(a)

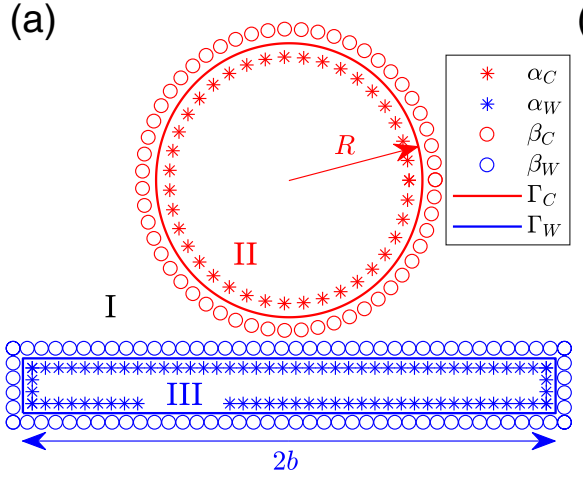

(b)

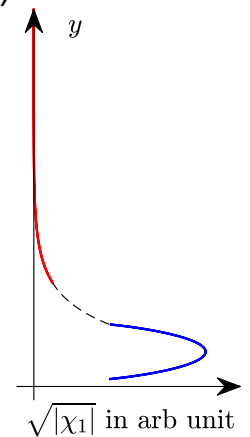

FIG. 13. Generalization of the MMP method to coupled graphene cavity-waveguide system. (a) Illustration of the MMP method, where two sets of boundaries, one of the cavity and another of the waveguide, divide the whole space into three regions: I, II, and III, corresponding to free space, cavity, and waveguide, respectively. Poles inside a domain are labeled with $\alpha$ and the outside poles are labeled with $\beta$. The cavity and waveguide boundaries are labeled as $\Gamma_{C}$ and $\Gamma_{W}$, respectively. (b) An MMP generated wave function, corresponding to the square root of $\left|\chi_{1}\right|$.

the transmission approaches the unity value. Figure 12(c1) shows the real part of $\psi_{1}$ calculated from the MMP method after normalization. The real part of $\psi_{2}$ is shown in Fig. 12(c2). The agreement is remarkable with small errors [Fig. 12(c3)], suggesting the reliability and accuracy of the MMP method as applied to an isolated graphene waveguide. While the calculation is illustrated for single mode operation, we have tested multimode operation and found that the MMP method is also applicable, albeit with slightly larger errors.

\section{APPENDIX C: MMP METHOD FOR THE COUPLED CAVITY-WAVEGUIDE SYSTEM}

For the simple situation where an electrical potential is applied to a single domain in the free space, by the MMP method [62-66], one distributes two sets of fictitious poles: one inside the domain another outside, where the wave function inside (outside) the domain is calculated as the contributions of all the poles outside (inside). Our coupled cavity-waveguide system is more complicated, as it contains two electrically generated, separate domains in the free space. Here we generalize the single domain MMP method to our coupled system, where the general setting is shown Fig. 13.

Similar to the setting of scattering from a single domain, we let $k_{1}=|\epsilon|, k_{2}=\left|\epsilon-V_{2}\right|$, and $k_{3}=\left|\epsilon-V_{3}\right|$, with the respective band indices $\tau_{1}=\operatorname{sign}(\epsilon), \tau_{2}=\operatorname{sign}\left(\epsilon-V_{2}\right)$, and $\tau_{3}=\operatorname{sign}\left(\epsilon-V_{3}\right)$. There are two boundaries: $\Gamma_{C}$ and $\Gamma_{W}$, each enclosing a domain, and multiple sets of poles are placed both inside and outside each domain. As shown in Fig. 13, altogether there are four types of poles: $\alpha_{C}, \beta_{C}, \alpha_{W}$, and $\beta_{W}$, with the respective numbers $N_{\alpha_{C}}, N_{\beta_{C}}, N_{\alpha_{W}}$, and $N_{\beta_{W}}$. We use the label $m_{\alpha_{C}} \in\left[1, N_{\alpha_{C}}\right]$ to denote each set of poles.
In free space, the wave function is determined by all the poles inside the waveguide and cavity domain:

$$
\begin{aligned}
\Psi^{(\mathrm{I})}(\mathbf{r})= & \sum_{m_{\alpha_{C}}} \sum_{l} G_{l}^{m_{\alpha_{C}}}\left(\begin{array}{c}
H_{l}^{(1)}\left(k_{1} d_{m_{\alpha_{C}}}\right) \\
i \tau_{1} H_{l+1}^{(1)}\left(k_{1} d_{m_{\alpha_{C}}}\right)
\end{array}\right) e^{i l l_{m_{\alpha_{C}}}} \\
& +\sum_{m_{\alpha_{W}}} \sum_{l} G_{l}^{m_{\alpha_{W}}}\left(\begin{array}{c}
H_{l}^{(1)}\left(k_{1} d_{m_{\alpha_{W}}}\right) \\
i \tau_{1} H_{l+1}^{(1)}\left(k_{1} d_{m_{\alpha_{W}}}\right)
\end{array}\right) e^{i l l_{m_{\alpha_{C}}},}
\end{aligned}
$$

where

$$
\begin{aligned}
d_{m_{\tau}} \equiv\left|\mathbf{d}_{m_{\tau}}\right| & =\left|\mathbf{r}-\mathbf{r}_{m_{\tau}}\right| \text { and } \theta_{m_{\tau}}=\operatorname{Angle}\left(\mathbf{r}-\mathbf{r}_{m_{\tau}}\right), \\
\tau & \in\left\{\alpha_{C}, \beta_{C}, \alpha_{W}, \beta_{W}\right\}, \\
m_{\tau} & \in\left[1, N_{\tau}\right],
\end{aligned}
$$

$G_{l}^{m_{\tau}}$ is the expansion coefficient for the $m_{\tau}$ th pole with the momentum index $l, \mathbf{r}_{\alpha_{C}}$ denotes the position for a pole inside the cavity, and $d_{m_{\alpha_{W}}}$ and $\theta_{m_{\alpha_{W}}}$ are the similarly defined quantities for the waveguide.

In the cavity (region II), the wave function is given by one subset of poles in free space (those near the cavity) as

$$
\Psi^{(\mathrm{II})}(\mathbf{r})=\sum_{m_{\beta_{C}}} \sum_{l} G_{l}^{\beta_{C}}\left(\begin{array}{l}
H_{l}^{(1)}\left(d_{m_{\beta_{C}}}\right) \\
H_{l+1}^{(1)}\left(d_{m_{\beta_{C}}}\right)
\end{array}\right) e^{i l \theta_{m_{\beta_{C}}}} .
$$

Similarly, in the waveguide (region III), the wave function is given by another subset of nearby poles in free space as

$$
\Psi^{(\mathrm{III})}(\mathbf{r})=\sum_{m_{\beta_{W}}} \sum_{l} G_{l}^{m_{\beta_{W}}}\left(\begin{array}{l}
H_{l}^{(1)}\left(d_{m_{\beta_{W}}}\right) \\
H_{l+1}^{(1)}\left(d_{m_{\beta_{W}}}\right)
\end{array}\right) e^{i l l_{m_{\beta_{W}}}} .
$$

With $\chi$ being the incident wave, inside the cavity we have

$$
\chi(\mathbf{r})=\left.\mathcal{N}_{\chi}\left(\begin{array}{c}
1 \\
\tau_{3}
\end{array}\right) \exp \left(i k_{x} x\right) \exp \left(-\alpha_{2}|y+d+a|\right)\right|_{\mathbf{r} \in \Gamma_{C}} .
$$

In the waveguide, we have

$$
\chi(\mathbf{r})=\left.\left(\begin{array}{c}
1 \\
\tau_{3}
\end{array}\right) \exp \left(i k_{x} x\right) \cos \left(\alpha_{1}|y+d+a|\right)\right|_{\mathbf{r} \in \Gamma_{W}} .
$$

To carry out normalization, we make use of the fact that the wave functions in the cavity and in the waveguide are coupled with each other by evanescent waves, as shown in Fig. 13. We have

$$
\mathcal{N}_{\chi}=\frac{\cos \left(\alpha_{1} a\right)}{\exp \left(-\alpha_{2} a\right)}
$$

This wave function is still not normalized. To complete the normalization, the free space solution is needed. Note that 
the total wave function must be continuous at the boundaries. Introducing the notation $\Psi=\left[\psi_{1}, \psi_{2}\right]^{T}$, we have

$$
\begin{aligned}
\left.\left(\Psi^{(\mathrm{II})}(\mathbf{r})+\chi(\mathbf{r})\right)\right|_{\mathbf{r} \in \Gamma_{C}} & =\left.\Psi^{(\mathrm{I})}(\mathbf{r})\right|_{\mathbf{r} \in \Gamma_{C}}, \\
\left.\left(\Psi^{(\mathrm{III})}(\mathbf{r})+\chi(\mathbf{r})\right)\right|_{\mathbf{r} \in \Gamma_{W}} & =\left.\Psi^{(\mathrm{I})}(\mathbf{r})\right|_{\mathbf{r} \in \Gamma_{W}} .
\end{aligned}
$$

The numbers of poles inside the cavity, inside the waveguide, outside but near the cavity, outside but in the vicinity of the waveguide are $N_{\alpha_{C}}, N_{\alpha_{W}}, N_{\beta_{C}}$, and $N_{\beta_{W}}$, respectively. At the cavity boundary, we choose $N_{\Gamma_{C}}$ discrete points. Similarly, we choose $N_{\Gamma_{W}}$ discrete points at the waveguide boundary. From each pole, the range of the momentum quantum number is $l \in[-L, L]$, with $N_{L} \equiv 2 L+1$.

The boundary condition can be written as a matrix equation

$$
\mathbb{M} * \mathbf{G}=\mathbf{Y} \text {. }
$$

For $\mathbb{M}$, we expand the four sets of poles to get

$$
\mathbb{M}=\left(\begin{array}{c|c|c|c}
\mathbb{A} \alpha C^{(\mathrm{II})} & \mathbb{A} \alpha W^{(\mathrm{II})} & -\mathbb{A} \beta C^{(\mathrm{II})} & \mathbb{Z}_{\beta W}^{(\mathrm{II})} \\
\hline \mathbb{B} \alpha C^{(\mathrm{II})} & \mathbb{B} \alpha W^{(\mathrm{II})} & -\mathbb{B} \beta C^{(\mathrm{II})} & \mathbb{Z}_{\beta W}^{(\mathrm{II})} \\
\hline \mathbb{A} \alpha C^{(\mathrm{III})} & \mathbb{A} \alpha W^{(\mathrm{III})} & \mathbb{Z}_{\beta C}^{(\mathrm{III})} & -\mathbb{A} \beta W^{(\mathrm{III})} \\
\hline \mathbb{B} \alpha C^{(\mathrm{III})} & \mathbb{B} \alpha W^{(\mathrm{III})} & \mathbb{Z}_{\beta C}^{(\mathrm{III})} & -\mathbb{B} \beta W^{(\mathrm{III})}
\end{array}\right),
$$

which can be expanded as $\mathbb{A}$ and $\mathbb{B}$, representing the magnitude of the first and second spinor component approaching the boundary, respectively. For example, $\mathbb{A} \alpha C^{(\mathrm{II})}$ denotes the poles inside the cavity $(\alpha C)$ reaching boundary II, with the associated matrix dimension $N_{\Gamma_{C}} \times N_{L} N_{\alpha_{C}}$. Explicitly, we have

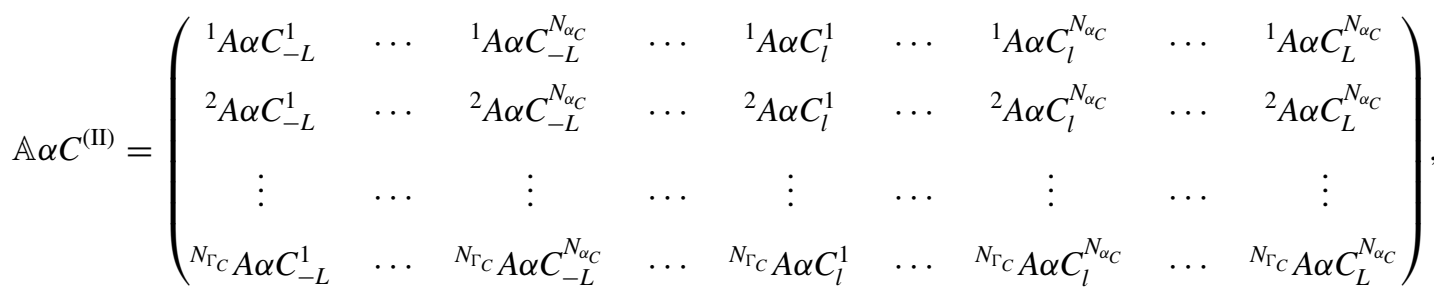

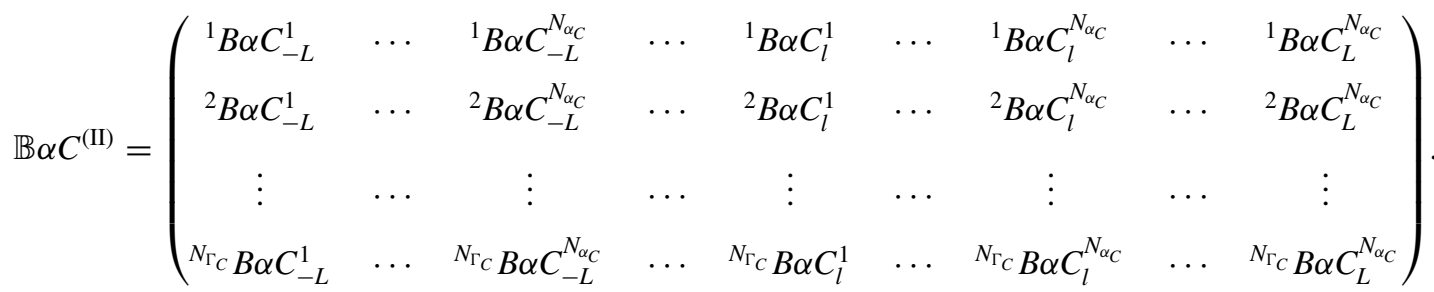

For ${ }^{i_{1}} A \alpha C_{i_{3}}^{i_{2}}, i_{1} \in\left[1, N_{\Gamma_{C}}\right]$ denotes the set of discrete boundary points, $i_{2} \in\left[1, N_{\alpha_{C}}\right]$ is the poles number, and $i_{3} \in[-L, L]$ is the momentum index. For example, we have

$$
\begin{aligned}
{ }^{i_{1}} A \alpha C_{i_{3}}^{i_{2}} & =H_{i_{3}}^{(1)}\left(k_{1} d_{i_{1} i_{2}}\right) \exp \left(i i_{3} \theta_{i_{1} i_{2}}\right), \\
{ }^{i_{1}} B \alpha C_{i_{3}}^{i_{2}} & =\tau_{1} H_{i_{3}+1}^{(1)}\left(k_{1} d_{i_{1} i_{2}}\right) \exp \left[i\left(i_{3}+1\right) \theta_{i_{1} i_{2}}\right], \\
d_{i_{1} i_{2}} & \equiv\left|\mathbf{d}_{i_{1} i_{2}}\right|=\left|\mathbf{r}_{i_{1}}-\mathbf{r}_{i_{2}}\right|, \\
\theta_{i_{1} i_{2}} & =\operatorname{Angle}\left(\mathbf{r}_{i_{1}}-\mathbf{r}_{i_{2}}\right) .
\end{aligned}
$$

The quantity $\mathbb{Z}_{\beta W}^{(\mathrm{II})}$ is the zero matrix of dimension $N_{\Gamma_{C}} \times N_{L} N_{\beta W}$. This means only poles outside but near the cavity contribute to the wave function inside the cavity.

The quantity $\mathbf{G}$ contains the unknown coefficients:

$$
\mathbf{G}=\left[\mathbf{G}^{\alpha_{C}}, \mathbf{G}^{\alpha_{W}}, \mathbf{G}^{\beta_{C}}, \mathbf{G}^{\beta_{W}}\right]^{T},
$$

where $\mathbf{G}^{\tau}$ denotes the coefficients associated with poles at $\tau$, for $\tau \in\left\{\alpha_{C}, \beta_{C}, \alpha_{W}, \beta_{W}\right\}$. Taking the first one as an example, we have

$$
\mathbf{G}^{\alpha_{C}}=\left(\begin{array}{c}
G_{-L}^{1} \\
\vdots \\
G_{l}^{1} \\
G_{l}^{2} \\
\vdots \\
G_{l}^{N_{\alpha_{C}}} \\
\vdots \\
G_{L}^{N_{\alpha_{C}}}
\end{array}\right)_{N_{\alpha_{C}} \times 1}
$$

The quantity $\mathbf{Y}$ denotes the incident wave given by

$$
\mathbf{Y}=\left[\chi_{1}^{\Gamma_{C}}, \chi_{2}^{\Gamma_{C}}, \chi_{1}^{\Gamma_{W}}, \chi_{2}^{\Gamma_{W}}\right]^{T} .
$$

For $\chi_{i_{2}}^{i_{1}}, i_{1} \in\left\{1, \Gamma_{W}\right\}$ indicates the discrete boundary points and $i_{2} \in\{1,2\}$ denotes the first and second spinor component. For example, we have

$$
\chi_{1}^{\Gamma_{C}}=\left(\begin{array}{c}
\chi_{1}^{1} \\
\chi_{1}^{2} \\
\vdots \\
\chi_{1}^{N_{\Gamma_{C}}}
\end{array}\right)_{N_{\Gamma_{C}} \times 1}
$$


(a1)

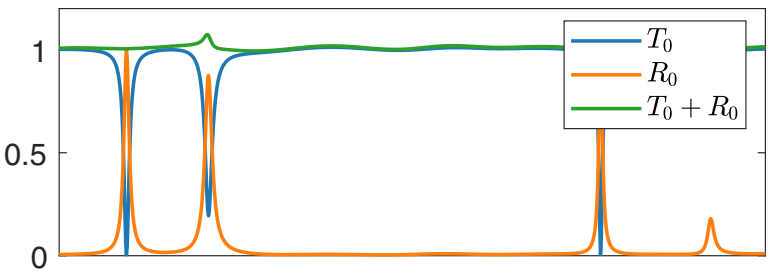

(a2)
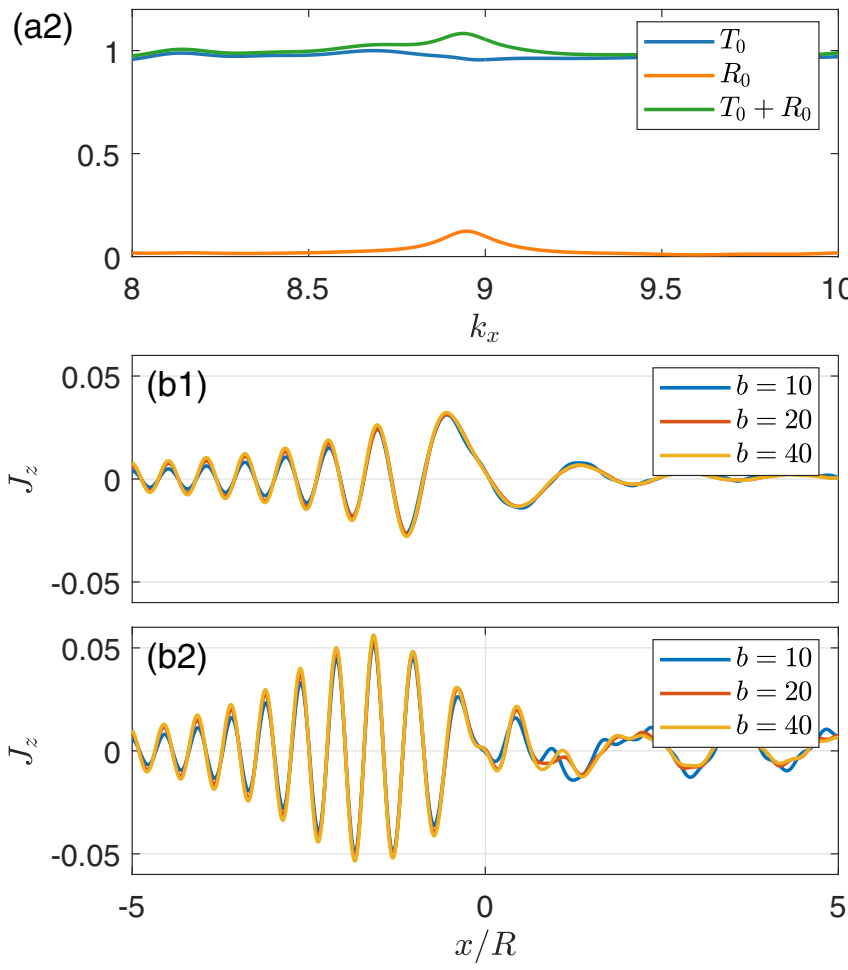

FIG. 14. Validation of the MMP method as applied to the coupled cavity-waveguide system. (a1) Transmission $T_{0}$ and reflection $R_{0}$ vs the input energy for the circular cavity, which are calculated from Eqs. (D1) and (D2), respectively. The deviation of the value of $T_{0}+R_{0}$ from unity is the result of a finite truncation of the waveguide in the propagating direction. (a2) The corresponding plot for the chaotic cavity with the shape of Africa. In both cases, the relative error is smaller than $10 \%$. (b1) The $z$ component of the pseudospin polarization for $k_{x} R=8.4231$ for the circular cavity, with parameter values the same as in Figs. 7(a)-7(c). The results for different values of $b$ converge. (b2) The corresponding plot for the deformed cavity for $k_{x} R=8.9623$ with the same parameter values as in Figs. 7(d)7 (f). There is also convergence for different values of the truncation length $b$.

with

$$
\chi_{i 1}^{i 2}=\chi_{i 1}\left(\mathbf{r}_{i 2}\right)
$$

We solve the equations by using the pseudoinverse method: $\mathbb{E}=\operatorname{pinv}(\mathbb{M}) \times \mathbf{Y}$. The error can be estimated as

$$
\mathrm{SSE}=\frac{\|\mathbb{M} * \mathbf{G}-\mathbf{Y}\|}{\|\mathbf{Y}\|} .
$$

In all calculations, we have $\mathrm{SSE}<2 \%$.

\section{APPENDIX D: VALIDATION OF MMP METHOD}

An ideal waveguide has an infinite length, for which the transmission is unity. With a finite truncation, the transmission value can deviate from unity, which constitutes the main error source of the MMP method. As shown in Fig. 12(b), the free space transmission approaches one as the truncation length $b$ is increased. We validate the MMP method as applied to the coupled cavity-waveguide system through the following steps.

\section{Transmission and reflection}

Since there are no intrinsic sources for the coupled system, after normalization, the transmission $T_{0}$ and reflection $R_{0}$ must satisfy $T_{0}+R_{0}=1$. For the transmission, we have

$$
T_{0}=J_{x}^{\text {out }} / J_{x}^{\text {out-Free }},
$$

where $J_{x}^{\text {out }}=\iint j_{x} d x d y$ and the integration is over the waveguide region beyond the coupling region directly below the cavity. The denominator is the integral of the corresponding free space solution. For reflection, we have

$$
R_{0}=1-J_{x}^{\text {in }} / J_{x}^{\text {in-Free }},
$$

where $J_{x}^{\text {in }}=\iint j_{x} d x d y$ and integration region is one in the waveguide before the coupling region. The denominator is the integral of the corresponding free space solution. While ideally, the integration range should be infinite, we find that carrying out the integral several cavity sizes away can give accurate solutions.

Figures 14(a1) and 14(a2) show $T_{0}+R_{0}$ versus the input energy $k_{x}$ for the circular and deformed cavities, respectively. Away from Fano resonance, we have $T_{0}+R_{0}=1$. At the Fano resonance point, the error is relatively large but is less than $10 \%$.

\section{Truncation error}

A finite truncation in $x$ will induce errors. However, in the $y$ and $z$ directions, no truncation is necessary. Figures 14(b1) and 14(b2) show the $z$ component of the pseudospin current $J_{z}$ for different values of $b$ for the circular and deformed cavities, respectively. We observe a good convergence.

[3] A. H. Castro Neto, F. Guinea, N. M. R. Peres, K. S. Novoselov, and A. K. Geim, The electronic properties of graphene, Rev. Mod. Phys. 81, 109 (2009).

[4] T. Wehling, A. M. Black-Schaffer, and A. V. Balatsky, Dirac materials, Adv. Phys. 63, 1 (2014).

[5] J. Wang, S. Deng, Z. Liu, and Z. Liu, The rare twodimensional materials with dirac cones, Natl. Sci. Rev. 2, 22 (2015). 
[6] P. San-Jose, E. Prada, E. McCann, and H. Schomerus, Pseudospin Valve in Bilayer Graphene: Towards Graphene-Based Pseudospintronics, Phys. Rev. Lett. 102, 247204 (2009).

[7] D. Pesin and A. H. MacDonald, Spintronics and pseudospintronics in graphene and topological insulators, Nat. Mater. 11, 409 (2012).

[8] W. Han, R. K. Kawakami, M. Gmitra, and J. Fabian, Graphene spintronics, Nat. Nanotech. 9, 794 (2014).

[9] A. Georgi, P. Nemes-Incze, R. Carrillo-Bastos, D. Faria, S. Viola Kusminskiy, D. Zhai, M. Schneider, D. Subramaniam, T. Mashoff, N. M. Freitag et al., Tuning the pseudospin polarization of graphene by a pseudomagnetic field, Nano Lett. 17, 2240 (2017).

[10] D. Frustaglia, M. Hentschel, and K. Richter, Quantum Transport in Nonuniform Magnetic Fields: Aharonov-Bohm Ring as A Spin Switch, Phys. Rev. Lett. 87, 256602 (2001).

[11] M. Johnson, Bipolar spin switch, Science 260, 320 (1993).

[12] A. I. Tartakovskii, T. Wright, A. Russell, V. I. Fal'ko, A. B. Van'kov, J. Skiba-Szymanska, I. Drouzas, R. S. Kolodka, M. S. Skolnick, P. W. Fry, A. Tahraoui, H.-Y. Liu, and M. Hopkinson, Nuclear Spin Switch in Semiconductor Quantum Dots, Phys. Rev. Lett. 98, 026806 (2007).

[13] I. Krivorotov, N. Emley, J. Sankey, S. Kiselev, D. Ralph, and R. Buhrman, Time-domain measurements of nanomagnet dynamics driven by spin-transfer torques, Science 307, 228 (2005).

[14] S. Kaka, M. R. Pufall, W. H. Rippard, T. J. Silva, S. E. Russek, and J. A. Katine, Mutual phase-locking of microwave spin torque nano-oscillators, Nature (London) 437, 389 (2005).

[15] F. Guinea, M. I. Katsnelson, and A. K. Geim, Energy gaps and a zero-field quantum Hall effect in graphene by strain engineering, Nat. Phys. 6, 30 (2010).

[16] J. Yan, L. Wu, R. Ma, S. Zhu, C. Bian, J. Ma, Q. Huan, L. Bao, J. Mao, S. Du et al., Substrate, a choice of engineering the pseudospin in graphene, 2D Mater. 6, 045050 (2019).

[17] J. Jung, F. Zhang, Z. Qiao, and A. H. MacDonald, Valley-Hall kink and edge states in multilayer graphene, Phys. Rev. B 84, 075418 (2011).

[18] M. Zarenia, O. Leenaerts, B. Partoens, and F. M. Peeters, Substrate-induced chiral states in graphene, Phys. Rev. B 86, 085451 (2012).

[19] J. Li, K. Wang, K. J. McFaul, Z. Zern, Y. Ren, K. Watanabe, T. Taniguchi, Z. Qiao, and J. Zhu, Gate-controlled topological conducting channels in bilayer graphene, Nat. Nanotech. 11, 1060 (2016).

[20] S. Fan, Sharp asymmetric line shapes in side-coupled waveguide-cavity systems, Appl. Phys. Lett. 80, 908 (2002).

[21] A. E. Miroshnichenko, S. Flach, and Y. S. Kivshar, Fano resonances in nanoscale structures, Rev. Mod. Phys. 82, 2257 (2010).

[22] A. Chiasera, Y. Dumeige, P. Feron, M. Ferrari, Y. Jestin, G. Nunzi Conti, S. Pelli, S. Soria, and G. C. Righini, Spherical whispering-gallery-mode microresonators, Laser Photon. Rev. 4, 457 (2010).

[23] H. Cao and J. Wiersig, Dielectric microcavities: Model systems for wave chaos and non-Hermitian physics, Rev. Mod. Phys. 87, 61 (2015).

[24] F.-M. Zhang, Y. He, and X. Chen, Guided modes in graphene waveguides, Appl. Phys. Lett. 94, 212105 (2009).
[25] R. R. Hartmann, N. J. Robinson, and M. E. Portnoi, Smooth electron waveguides in graphene, Phys. Rev. B 81, 245431 (2010).

[26] N. Myoung, G. Ihm, and S. J. Lee, Magnetically induced waveguide in graphene, Phys. Rev. B 83, 113407 (2011).

[27] R. R. Hartmann and M. E. Portnoi, Quasi-exact solution to the dirac equation for the hyperbolic-secant potential, Phys. Rev. A 89, 012101 (2014).

[28] J. Williams, T. Low, M. Lundstrom, and C. Marcus, Gatecontrolled guiding of electrons in graphene, Nat. Nanotech. 6, 222 (2011).

[29] P. Rickhaus, M.-H. Liu, P. Makk, R. Maurand, S. Hess, S. Zihlmann, M. Weiss, K. Richter, and C. Schönenberger, Guiding of electrons in a few-mode ballistic graphene channel, Nano Lett. 15, 5819 (2015).

[30] Y. Zhao, J. Wyrick, F. D. Natterer, J. F. Rodriguez-Nieva, C. Lewandowski, K. Watanabe, T. Taniguchi, L. S. Levitov, N. B. Zhitenev, and J. A. Stroscio, Creating and probing electron whispering-gallery modes in graphene, Science 348, 672 (2015).

[31] J. Lee, D. Wong, J. Velasco Jr, J. F. Rodriguez-Nieva, S. Kahn, H.-Z. Tsai, T. Taniguchi, K. Watanabe, A. Zettl, F. Wang et al., Imaging electrostatically confined Dirac fermions in graphene quantum dots, Nat. Phys. 12, 1032 (2016).

[32] C. Gutiérrez, L. Brown, C.-J. Kim, J. Park, and A. N. Pasupathy, Klein tunneling and electron trapping in nanometrescale graphene quantum dots, Nat. Phys. 12, 1069 (2016).

[33] J. Velasco Jr, L. Ju, D. Wong, S. Kahn, J. Lee, H.-Z. Tsai, C. Germany, S. Wickenburg, J. Lu, T. Taniguchi et al., Nanoscale control of rewriteable doping patterns in pristine graphene/boron nitride heterostructures, Nano Lett. 16, 1620 (2016).

[34] F. Ghahari, D. Walkup, C. Gutiérrez, J. F. Rodriguez-Nieva, Y. Zhao, J. Wyrick, F. D. Natterer, W. G. Cullen, K. Watanabe, T. Taniguchi et al., An on/off Berry phase switch in circular graphene resonators, Science 356, 845 (2017).

[35] D. S. Novikov, Elastic scattering theory and transport in graphene, Phys. Rev. B 76, 245435 (2007).

[36] R. L. Heinisch, F. X. Bronold, and H. Fehske, Mie scattering analog in graphene: Lensing, particle confinement, and depletion of Klein tunneling, Phys. Rev. B 87, 155409 (2013).

[37] J.-S. Wu et al., Scattering of two-dimensional massless Dirac electrons by a circular potential barrier, Phys. Rev. B 90, 235402 (2014).

[38] C. A. Downing, A. R. Pearce, R. J. Churchill, and M. E. Portnoi, Optimal traps in graphene, Phys. Rev. B 92, 165401 (2015).

[39] M. V. Berry and R. Mondragon, Neutrino billiards: timereversal symmetry-breaking without magnetic fields, Proc. R. Soc. London A 412, 53 (1987).

[40] H. Xu, L. Huang, Y.-C. Lai, and C. Grebogi, Chiral Scars in Chaotic Dirac Fermion Systems, Phys. Rev. Lett. 110, 064102 (2013).

[41] Z.-Q. Fu, Y.-T. Pan, J.-J. Zhou, D.-L. Ma, Y. Zhang, J.-B. Qiao, H. Liu, H. Jiang, and L. He, Relativistic artificial molecules realized by two coupled graphene quantum dots, arXiv:1908.06580.

[42] M. Sadrara and M. F. Miri, Dirac electron scattering from a cluster of electrostatically defined quantum dots in graphene, Phys. Rev. B 99, 155432 (2019). 
[43] S. Tomsovic and D. Ullmo, Chaos-assisted tunneling, Phys. Rev. E 50, 145 (1994).

[44] F. Leyvraz and D. Ullmo, The level splitting distribution in chaos-assisted tunnelling, J. Phys. A: Math. Gen. 29, 2529 (1996).

[45] J. Zakrzewski, D. Delande, and A. Buchleitner, Ionization via chaos assisted tunneling, Phys. Rev. E 57, 1458 (1998).

[46] D. A. Steck, W. H. Oskay, and M. G. Raizen, Observation of chaos-assisted tunneling between islands of stability, Science 293, 274 (2001).

[47] A. Mouchet, C. Miniatura, R. Kaiser, B. Grémaud, and D. Delande, Chaos-assisted tunneling with cold atoms, Phys. Rev. E 64, 016221 (2001).

[48] E. E. Narimanov and V. A. Podolskiy, Chaos-assisted tunneling and dynamical localization in dielectric microdisk resonators, IEEE J. Sel. Top. Quantum Electron. 12, 40 (2006).

[49] A. Relaño, Chaos-Assisted Tunneling and $1 / f^{\alpha}$ Spectral Fluctuations in the Order-Chaos Transition, Phys. Rev. Lett. 100, 224101 (2008).

[50] A. Bäcker, R. Ketzmerick, S. Löck, and L. Schilling, Regularto-Chaotic Tunneling Rates Using A Fictitious Integrable System, Phys. Rev. Lett. 100, 104101 (2008).

[51] S. Löck, A. Bäcker, R. Ketzmerick, and P. Schlagheck, Regularto-Chaotic Tunneling Rates: From the Quantum to the Semiclassical Regime, Phys. Rev. Lett. 104, 114101 (2010).

[52] Q. Song, L. Ge, B. Redding, and H. Cao, Channeling Chaotic Rays Into Waveguides for Efficient Collection of Microcavity Emission, Phys. Rev. Lett. 108, 243902 (2012).

[53] M.-W. Kim, S. Rim, C.-H. Yi, and C.-M. Kim, Chaos-assisted tunneling in a deformed microcavity laser, Opt. Express 21, 32508 (2013).

[54] S. Gehler, S. Löck, S. Shinohara, A. Bäcker, R. Ketzmerick, U. Kuhl, and H.-J. Stöckmann, Experimental Observation of Resonance-Assisted Tunneling, Phys. Rev. Lett. 115, 104101 (2015).

[55] N. Mertig, J. Kullig, C. Löbner, A. Bäcker, and R. Ketzmerick, Perturbation-free prediction of resonance-assisted tunneling in mixed regular-chaotic systems, Phys. Rev. E 94, 062220 (2016).

[56] E. V. H. Doggen, B. Georgeot, and G. Lemarié, Chaos-assisted tunneling in the presence of Anderson localization, Phys. Rev. E 96, 040201(R) (2017).

[57] X. Jiang, L. Shao, S.-X. Zhang, X. Yi, J. Wiersig, L. Wang, Q. Gong, M. Lončar, L. Yang, and Y.-F. Xiao, Chaos-assisted broadband momentum transformation in optical microresonators, Science 358, 344 (2017).

[58] P. Yu, T. Hu, H. Qiu, F. Ge, H. Yu, X. Jiang, and J. Yang, Fano resonances in ultracompact waveguide Fabry-Perot resonator side-coupled lossy nanobeam cavities, Appl. Phys. Lett. 103, 091104 (2013).

[59] N. Dabidian, I. Kholmanov, A. B. Khanikaev, K. Tatar, S. Trendafilov, S. H. Mousavi, C. Magnuson, R. S. Ruoff, and G. Shvets, Electrical switching of infrared light using graphene integration with plasmonic Fano resonant metasurfaces, ACS Photonics 2, 216 (2015).

[60] C. Argyropoulos, Enhanced transmission modulation based on dielectric metasurfaces loaded with graphene, Opt. Express 23, 23787 (2015).

[61] R. N. Patel, Z. Wang, W. Jiang, C. J. Sarabalis, J. T. Hill, and A. H. Safavi-Naeini, Single-Mode Phononic Wire, Phys. Rev. Lett. 121, 040501 (2018).
[62] Y. Leviatan and A. Boag, Analysis of electromagnetic scattering from dielectric cylinders using a multifilament current model, IEEE Trans. Antennas Propag. 35, 1119 (1987).

[63] M. G. Imhof, Multiple multipole expansions for elastic scattering, J. Acoust. Soc. Am. 100, 2969 (1996).

[64] D. I. Kaklamani and H. T. Anastassiu, Aspects of the method of auxiliary sources (MAS) in computational electromagnetics, IEEE Anten. Propag. Maga. 44, 48 (2002).

[65] E. Moreno, D. Erni, C. Hafner, and R. Vahldieck, Multiple multipole method with automatic multipole setting applied to the simulation of surface plasmons in metallic nanostructures, J. Opt. Soc. Am. A 19, 101 (2002).

[66] G. Tayeb and S. Enoch, Combined fictitious-sourcesscattering-matrix method, J. Opt. Soc. Am. A 21, 1417 (2004).

[67] E. Moreno, D. Erni, and C. Hafner, Modeling of discontinuities in photonic crystal waveguides with the multiple multipole method, Phys. Rev. E 66, 036618 (2002).

[68] H.-Y. Xu and Y.-C. Lai, Pseudospin-1 wave scattering that defies chaos Q-spoiling and Klein tunneling, Phys. Rev. B 99, 235403 (2019).

[69] H.-Y. Xu and Y.-C. Lai, Anomalous chiral edge states in spin-1 Dirac quantum dots, Phys. Rev. Res. 2, 013062 (2020).

[70] C.-Z. Wang, H.-Y. Xu, and Y.-C. Lai, Scattering of Dirac electrons from a skyrmion: Emergence of robust skew scattering, Phys. Rev. Res. 2, 013247 (2020).

[71] U. Fano, Effects of configuration interaction on intensities and phase shifts, Phys. Rev. 124, 1866 (1961).

[72] L. Huang, Y.-C. Lai, H.-G. Luo, and C. Grebogi, Universal formalism of Fano resonance, AIP Adv. 5, 017137 (2015).

[73] Y.-Z. Gu, S.-X. Zhang, F. Gao, Q.-H. Gong, and Y.-F. Xiao, Wave-scattering method for waveguide-microcavity coupling, J. Opt. Soc. B 35, 811 (2018).

[74] M. A. Popović, C. Manolatou, and M. R. Watts, Couplinginduced resonance frequency shifts in coupled dielectric multicavity filters, Opt. Express 14, 1208 (2006).

[75] X. Piao, S. Yu, J. Hong, and N. Park, Spectral separation of optical spin based on antisymmetric Fano resonances, Sci. Rep. 5, 16585 (2015).

[76] H. E. Tureci, H. G. L. Schwefel, P. Jacquod, and A. D. Stone, Modes of wave-chaotic dielectric resonators, Prog. Opt. 47, 75 (2005).

[77] J. H. Bardarson, M. Titov, and P. W. Brouwer, Electrostatic Confinement of Electrons in An Integrable Graphene Quantum Dot, Phys. Rev. Lett. 102, 226803 (2009).

[78] C.-Z. Wang, C.-D. Han, H.-Y. Xu, and Y.-C. Lai, Chaos-based Berry phase detector, Phys. Rev. B 99, 144302 (2019).

[79] C.-D. Han, H.-Y. Xu, and Y.-C. Lai, Electrical confinement in a spectrum of two-dimensional Dirac materials with classically integrable, mixed, and chaotic dynamics, Phys. Rev. Research 2, 013116 (2020).

[80] M. Viret, D. Vignoles, D. Cole, J. M. D. Coey, W. Allen, D. S. Daniel, and J. F. Gregg, Spin scattering in ferromagnetic thin films, Phys. Rev. B 53, 8464 (1996).

[81] M. Berry, Five momenta, Eur. J. Phys. 34, 1337 (2013).

[82] A. L. Friedman, O. M. van't Erve, C. H. Li, J. T. Robinson, and B. T. Jonker, Homoepitaxial tunnel barriers with functionalized graphene-on-graphene for charge and spin transport, Nat. Commun. 5, 3161 (2014). 
[83] M. V. Kamalakar, C. Groenveld, A. Dankert, and S. P. Dash, Long distance spin communication in chemical vapour deposited graphene, Nat. Commun. 6, 6766 (2015).

[84] T. Danz, A. Neff, J. H. Gaida, R. Bormann, C. Ropers, and S. Schäfer, Ultrafast sublattice pseudospin relaxation in graphene probed by polarization-resolved photoluminescence, Phys. Rev. B 95, 241412(R) (2017).

[85] H.-J. Stöckmann, Quantum Chaos: An Introduction (Cambridge University Press, New York, 2006).

[86] M. V. Berry, Regular and irregular semiclassical wavefunctions, J. Phys. A: Math. Gen. 10, 2083 (1977).

[87] M. V. Berry, Semiclassical theory of spectral rigidity, Proc. R. Soc. London A 400, 229 (1985).

[88] Y.-C. Lai, R. Blümel, E. Ott, and C. Grebogi, Quantum Manifestations of Chaotic Scattering, Phys. Rev. Lett. 68, 3491 (1992).

[89] R. Ketzmerick, Fractal conductance fluctuations in generic chaotic cavities, Phys. Rev. B 54, 10841 (1996).

[90] A. S. Sachrajda, R. Ketzmerick, C. Gould, Y. Feng, P. J. Kelly, A. Delage, and Z. Wasilewski, Fractal Conductance Fluctua- tions in A Soft-Wall Stadium and A Sinai Billiard, Phys. Rev. Lett. 80, 1948 (1998).

[91] B. Huckestein, R. Ketzmerick, and C. H. Lewenkopf, Quantum Transport through Ballistic Cavities: Soft vs Hard Quantum Chaos, Phys. Rev. Lett. 84, 5504 (2000).

[92] K. Clauß, M. J. Körber, A. Bäcker, and R. Ketzmerick, Resonance Eigenfunction Hypothesis for Chaotic Systems, Phys. Rev. Lett. 121, 074101 (2018).

[93] O. Zaitsev, D. Frustaglia, and K. Richter, Role of Orbital Dynamics in Spin Relaxation and Weak Antilocalization in Quantum Dots, Phys. Rev. Lett. 94, 026809 (2005).

[94] J. Wurm, K. Richter, and I.ç Adagideli, Edge effects in graphene nanostructures: Semiclassical theory of spectral fluctuations and quantum transport, Phys. Rev. B 84, 205421 (2011).

[95] C.-D. Han, C.-Z. Wang, H.-Y. Xu, D. Huang, and Y.-C. Lai, Decay of semiclassical massless Dirac fermions from integrable and chaotic cavities, Phys. Rev. B 98, 104308 (2018).

[96] F. W. Olver, D. W. Lozier, R. F. Boisvert, and C. W. Clark, NIST Handbook of Mathematical Functions (Cambridge university press, New York, NY, 2010). 